\title{
Phonon-assisted diffusion in the bcc phase of titanium and zirconium from first-principles
}

\author{
Sara Kadkhodaei* and Ali Davariashtiyani \\ University of Illinois at Chicago, Chicago, Illinois, USA
}

(Dated: June 14, 2021)

\begin{abstract}
Diffusion is the underlying mechanism for many complicated materials phenomena, and understanding it is basic to discovery of novel materials with desired physical and mechanical properties. Certain groups of solid phases, such as the bcc phase of IIIB and IVB metals and their alloys, which are only stable when they reach high enough temperatures and experience anharmonic vibration entropic effects, exhibit "anomalously fast diffusion". However, the underlying reason for the observed extraordinary fast diffusion is poorly understood and due to the existence of harmonic vibration instabilities in these phases the standard models fail to predict their diffusivity. Here, we indicate that the anharmonic phonon-phonon coupling effects can accurately describe the anomalously large macroscopic diffusion coefficients in the bcc phase of IVB metals, and therefore yield new understanding on the underlying mechanism for diffusion in these phases. We utilize temperature-dependent phonon analysis by combining ab initio molecular dynamics with lattice dynamics calculations to provide a new approach to use the transition state theory beyond the harmonic approximation. We validate the diffusivity predictions for the bcc phase of titanium and zirconium with available experimental measurements, while we show that predictions based on harmonic transition state theory severely underestimates diffusivity in these phases.
\end{abstract}

\section{INTRODUCTION}

Diffusion processes play a key role in the kinetics of many materials-related phenomena, such as microstructural evolution during materials processing, and discovery of materials with specific diffusion characteristics is a major effort in various research communities. Understanding mechanisms of diffusion and providing an $a$ priori predictive ability for diffusivity are essential for discovery of materials with specific diffusion characteristics and first-principles methods have become a common approach to predict diffusivity in various materials [115]. Existing computational frameworks based on the harmonic transition state theory $[16,17]$ rely on the assumption that the solid phase is metastable and there exists no mechanical instabilities in the system. This assumption impedes the use of standard models to predict diffusivity in many solid-state systems [18-29], including the $\beta$ phase of group IIIB and IVB metals and their alloys, where the system exhibits harmonic phonon instabilities yet is stabilized at high enough temperatures due to strongly anharmonic lattice vibration entropic effects. Moreover, $\beta$ phase of IIIB and IVB metals and their alloys exhibit "anomalously fast diffusion", several orders of magnitude higher than fcc metals and other bcc metals, at temperatures far below their melting point [30$36]$, yet the underlying reason for this anomaly is poorly understood.

A number of studies have proposed explanations for the anomalous fast diffusion in the bcc phase of IIIB and IVB metals [37-45]. While some earlier studies proposed mixed vacancy mechanisms similar to contribution

\footnotetext{
* To whom correspondence should be addressed; Email: sarakad@uic.edu
}

of divacancies jump or mobile defects in fcc metals as the basis of anomalous fast diffusion, later experimental evidence based on isotope effect measurements and neutron scattering in $\beta$ IIIB and IVB metals confirmed that the monovacancy mechanism is the predominant diffusion mechanism [31-33, 35, 46, 47]. Recently, Sangiovanni et al. [44] proposed a highly concerted stringlike atomic motion as the mechanism underlying anomalously large self-diffusivities in bcc Ti based on observations in an $a b$ initio molecular dynamics simulation. Other studies correlated the markedly fast diffusion to temperature-induced effects. For example, Sanchez and de Fontaine proposed a model which correlates diffusivity in $\beta$-Zr to the formation of the metastable $\omega$ phase (i.e., heterophase fluctuations between bcc and $\omega$ induced by anharmonic vibrations), in which the diffusion activation energy is assumed to be the formation free energy of $\omega$ embryo [38, 39]. Herzig and co-workers concluded that the observed anomalous fast self-diffusion corresponds to the softening of the LA $\frac{2}{3}\langle 111\rangle$ phonon mode [40-42], supported by observation of higher diffusivity in phases with stronger softening effects [40].

The contribution of this report is twofold; First we illustrate that while the monovacancy jump is the predominant mechanism for diffusion in the bcc phase of $\mathrm{Ti}$ and $\mathrm{Zr}$, it is promoted by the anharmonic phononphonon coupling effects, which underlies the observed anomalously fast diffusion. Second, we provide a new approach based on first-principles calculations that can successfully predict the macroscopic diffusion coefficient for the bcc phase of titanium and zirconium where the standard computational frameworks based on harmonic transition state theory fail. We have previously shown that for bcc IVB metals and their compounds (which are mechanically unstable), temperature-induced dynamical stabilization results in hopping of the system among lo- 
cal distortions of the lattice (i.e., local minima) in a way that the average atomic positions stay at bcc [22, 28]. These local minima are located along the trigonal path associated with the longitudinal $\mathrm{L} \frac{2}{3}\langle 111\rangle$ phonon eigenvector [22, 28]. Here we show that the coincidence of this dynamical hopping with the direction of vacancy jump along $\frac{1}{2}\langle 111\rangle$ nearest neighbor in bcc metals significantly promotes the macroscopic diffusivity. Our $a$ priori prediction is validated with available experimental diffusivity measurements. On the other hand, we indicate that predicting diffusivity based on harmonic lattice vibrations severely under-predicts this diffusivity. This further illustrates the significant role of anharmonic lattice vibration interactions in enhancing the diffusivity. Our findings shed light on understanding the fundamental origins of diffusion mechanism in entropy-stabilized phases.

\section{ANHARMONIC LATTICE VIBRATION EFFECTS WITHIN TRANSITION STATE THEORY}

The macroscopic diffusivity $D$, for a vacancy-mediated diffusion can be described in terms of the atomic jump distance and jump frequency [48, 49], as the following,

$$
D=\alpha C_{v} a^{2} \Gamma
$$

where $\alpha$ is a geometrical factor ( $\alpha=8 / 6$ for bcc), $a$ is the vacancy jump distance, $C_{v}$ is the equilibrium vacancy concentration, and $\Gamma$ is the successful vacancy jump frequency [48]. Within the transition state theory $[1,16,17,50]$, the defect jump frequency is expressed as $\Gamma=\nu^{*} \exp \left(-\Delta H_{m} / k_{B} T\right)$, where $\Delta H_{m}$ is the migration enthalpy. $k_{B}$ is the Boltzmann's constant and $T$ denotes temperature. $\nu^{*}$ is the effective prefactor frequency, which encompasses all vibrational effects in the diffusion process. Within the harmonic transition state theory (HTST), $\nu^{*}$ is expressed as the ratio of the product of normal vibration frequencies (i.e., harmonic phonon frequencies) of the initial state to that of the non-imaginary normal frequencies of the transition state, $\nu^{*}=\frac{\prod_{i=1}^{3 N} \nu_{i}}{\prod_{i=1}^{3 N-1} \nu_{i}^{\prime}}$, where $\nu$ and $\nu^{\prime}$ are the frequencies for the initial and transition configurations, respectively, for a system of $N$ atoms. The equilibrium monovacancy concentration, $C_{v}$, is calculated according to $C_{v}=\exp \left(-\frac{\Delta H_{v}-T \Delta S_{v}}{k_{B} T}\right)$, where $\Delta H_{v}$ and $\Delta S_{v}$ are enthalpy and entropy of monovacancy formation, respectively. The formation enthalpy and entropy are calculated according to $\Delta H_{v}=H^{t o t}(N-1)-\frac{N-1}{N} H^{t o t}(N)$ and $\Delta S_{v}=S^{\text {tot }}(N-1)-\frac{N-1}{N} S^{\text {tot }}(N)$, respectively. $H^{\text {tot }}(N-$ $1)$ and $S^{\text {tot }}(N-1)$ are the total enthalpy and entropy for a system with a monovacancy and $H^{\text {tot }}(N)$ and $S^{\text {tot }}(N)$ are the total enthalpy and entropy for the bulk phase, respectively. The vibration entropy for each system is obtained from $S_{v i b}=-k_{B} \int_{0}^{\infty} \ln \left[2 \sinh \left(\frac{\hbar \omega}{2 k_{B} T}\right)\right] g(\omega) d \omega$, where $\omega$ is the vibration frequency and $g(\omega)$ is phonon density of state (DOS).

For systems that exhibit harmonic phonon instabilities, such as the bcc phase of IVB metals, calculation of diffusivity according to harmonic phonon analysis becomes infeasible. More specifically, the existence of multiple imaginary $\nu$ and $\nu^{\prime}$ frequencies impedes the use of HTST to obtain $\nu^{*}$, and $S_{v i b}$ cannot be obtained based on imaginary phonon frequencies. In Fig. 1, the harmonic phonon dispersions for bcc $\mathrm{Ti}$ with a monovacancy at the initial and an intermediate state are obtained based on the density-functional theory (DFT) finite displacement approach, where the harmonic phonon dispersions exhibit phonon instabilities (similar plot for bcc $\mathrm{Zr}$ is presented in Fig. S5 in [51]). However, similar to other bcc IIIB and IVB metals, bcc $\mathrm{Ti}$ is stabilized at high enough temperature due to the entropic stabilization of the system arising from large amplitude anharmonic hopping among local distortions [18-22, 24, 26-28, 52-59]. We utilize two different variations of the self-consistent harmonic approximation (SCHA) approach, namely the self-consistent $a b$ initio lattice dynamics (SCAILD) [60$62]$ and the temperature dependent effective potential (TDEP) method [63, 64], to obtain the temperaturedependent phonon dispersion and DOS for well-defined equilibrium states, namely bulk bcc Ti and the bcc phase with a monovacancy (see section SI in [51] for more details). Fig. 2 compares the harmonic phonon dispersion (i.e., the zero-temperature phonon dispersion) of bcc Ti including phonon instabilities with finite-temperature phonon dispersions from $1300 \mathrm{~K}$ to $1600 \mathrm{~K}$ with no imaginary phonon frequencies, as expected due to dynamical stabilization of the phase (similar plot for bcc Zr is presented in Fig. S6 in [51]).

As illustrated in Fig. 2(b)-(d), there is a negative dip at $\xi=\frac{2}{3}$ on the $L\langle\xi \xi \xi\rangle$ phonon branch (depicted as the dashed line in Fig. 2(d)), which is indicative of the phonon softening effects. This phonon mode corresponds to the trigonal distortion in which two neighboring (111) planes move towards each other and results in the $\omega$ phase when they collapse (see Fig 2(e)). This atomic distortion coincides with the vacancy jump direction for the bcc phase. Accordingly, we assume that the effective prefactor frequency, $\nu^{*}(\mathrm{~T})$, which is the effective vibration frequency along the transition path, is equal to the temperature-dependent frequency of the $\mathrm{L} \frac{2}{3}\langle 111\rangle$ phonon mode for the bulk bcc phase.

The successful jump frequency, $\Gamma$, can then be obtained given the anharmonic effective frequency, $\nu^{*}$, and the activation free energy. To calculate the activation free energy in systems with harmonic phonon instabilities, there are some bottlenecks. Since the structure is mechanically unstable and only stabilizes at high temperatures, it cannot be relaxed according to zero-temperature forces to obtain the vacancy formation enthalpy. Additionally, common schemes such as the nudged elastic band (NEB) or dimer method [65-67] cannot be used to locate the transition state as they rely on the assumption that the 

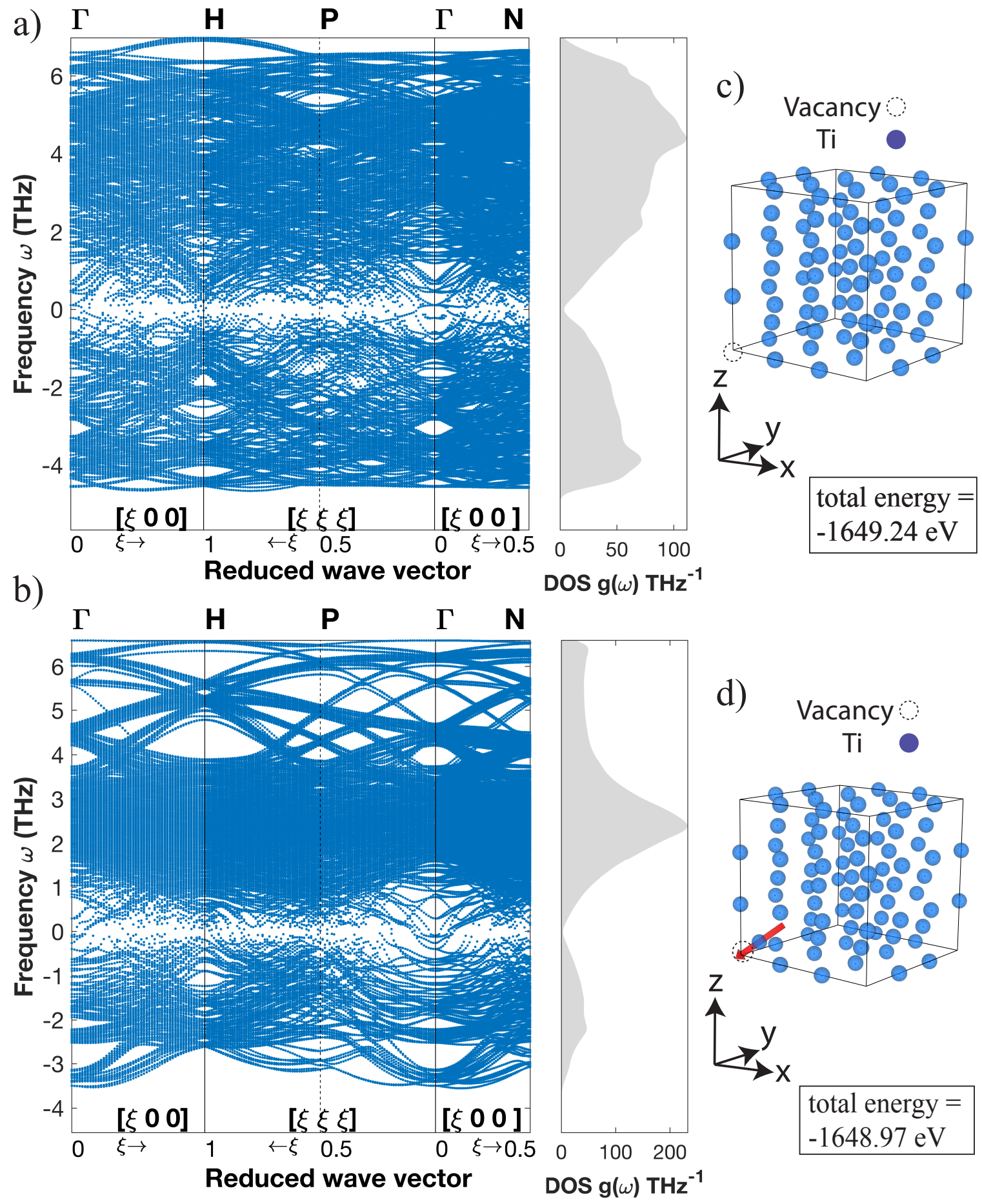

d)

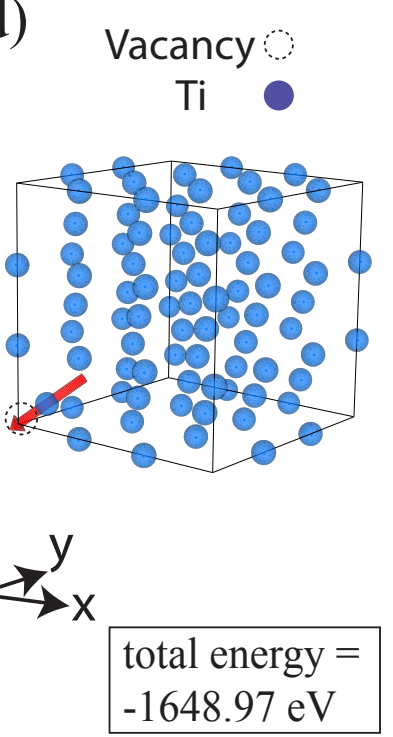

FIG. 1. DFT harmonic phonon dispersion and density of states (DOS) for a $6 \times 6 \times 6$ supercell of bcc titanium including 215 atoms and a monovacancy a) at the bcc site, and b) migrated half way to the nearest neighbor bcc site along [111] direction. Phonon instabilities are depicted as negative frequencies in the dispersion curves. The atomic configuration for the initial and intermediate states of vacancy diffusion are illustrated in c) and d), respectively. A conventional supercell is presented for visualization simplicity.

final and initial states of diffusion are metastable. Moreover, existence of imaginary phonon frequencies inhibits the calculation of vacancy formation entropy, $S_{v i b}$. To overcome these problem, we use the statistical average energy and pressure from NVT ab initio molecular dynamics (AIMD) simulations to obtain the enthalpy of the system at high temperature. In addition, vacancy formation entropy is obtained by comparing the temperature- 

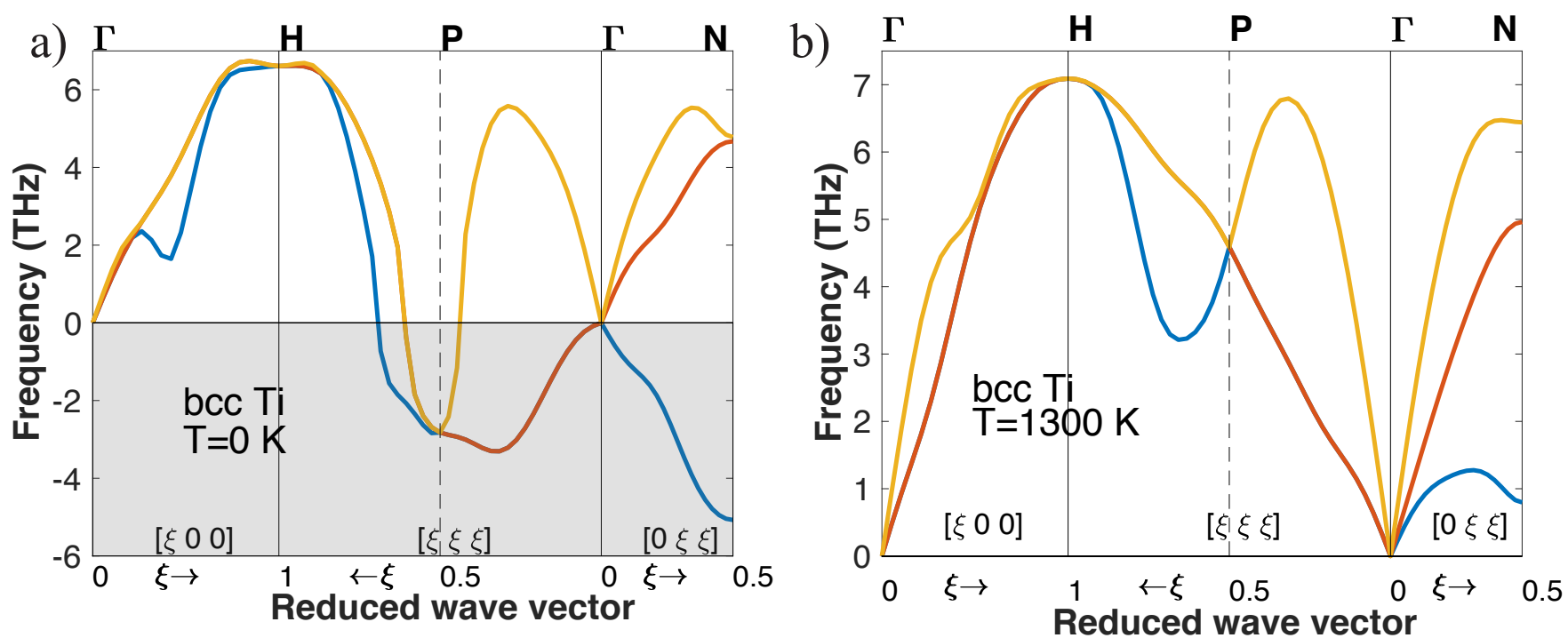

c)

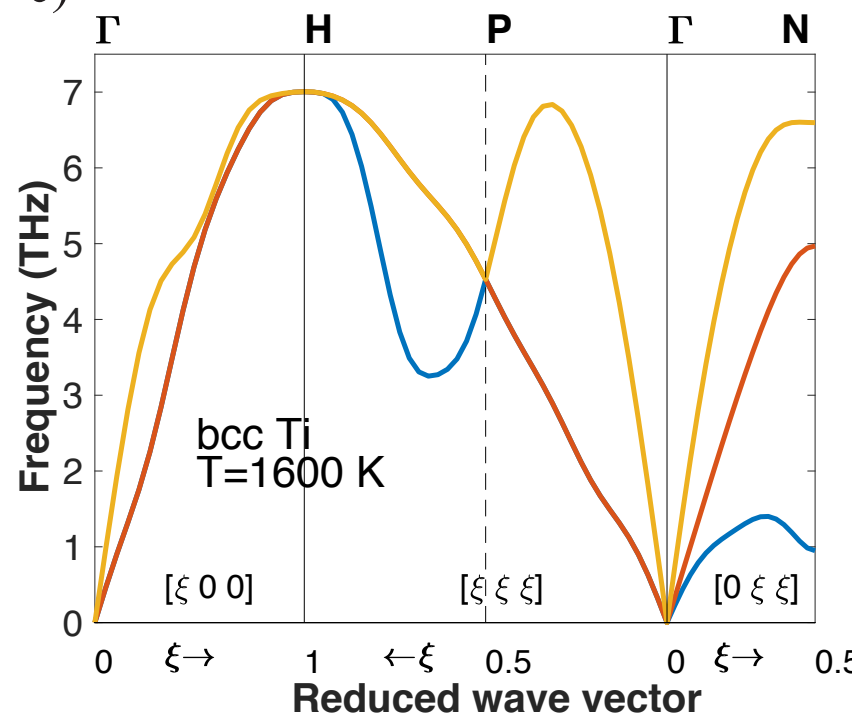

d)

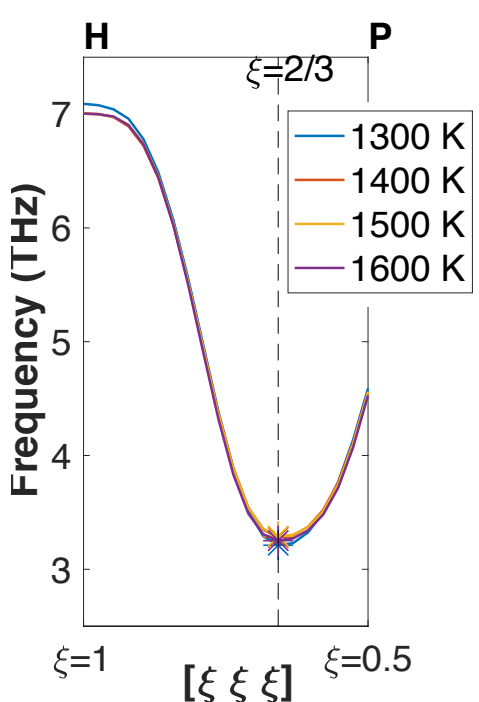

e)

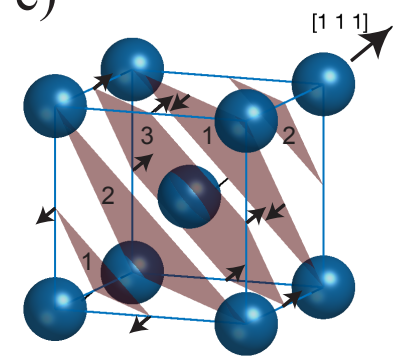

1231

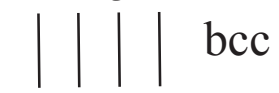

] $\mid[-\operatorname{bcc} \rightarrow \omega$

$1,321,3$

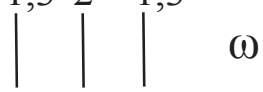

FIG. 2. Phonon dispersion for a bulk defect-free $6 \times 6 \times 6$ supercell of bcc titanium including 216 atoms at a) $0 \mathrm{~K}$, b) $1300 \mathrm{~K}$, and c) $1600 \mathrm{~K}$ obtained using the SCAILD method. d) The longitudinal phonon branch along the [ $\xi \xi \xi \xi$ reduced wave vector (between $\mathrm{H}$ and $\mathrm{P}$ high-symmetry points) from $1300 \mathrm{~K}$ to $1600 \mathrm{~K}$. The star marks in (d) represent the negative dip on the phonon branch. e) The trigonal distortion of (111) atomic planes along the [111] direction associates with the eigenvector of $\xi=\frac{2}{3}$ phonon mode along the $\Gamma-\mathrm{P}-\mathrm{H}$ branch. A complete collapse of atomic planes 1 and 3 results in the hexagonal $\omega$ phase.

dependent phonon frequencies and DOS for the bulk and defected systems. For the specific problem of bcc IVB metals, we assume that an interpolated state between the initial and final states (see Fig. 1(d)) is a reasonable approximation for the transition state. Accordingly, all the parameters needed for diffusivity calculation in Eq. 1 can be obtained as temperature-dependent parameters that effectively account for phonon-phonon coupling effects:

$$
\begin{aligned}
D & =\alpha a^{2} C_{v} \Gamma \\
& =\alpha a^{2} \nu^{*}(T) \exp \left(\frac{-\Delta H_{m}-\Delta H_{v}(T)}{k_{B} T}\right) \exp \left(\frac{-\Delta S_{v}(T)}{k_{B}}\right) .
\end{aligned}
$$

\section{APPLICATION TO THE BCC PHASE OF IVB METALS}

We applied the proposed methodology to obtain macroscopic diffusion coefficient for the bcc phase of titanium and zirconium. We calculate the migration enthalpy, $\Delta H_{m}$, as the DFT energy difference between the intermediate state and the initial state (represented in Fig. 1(c)-(d)). Note that we neglect the temperatureinduced phonon-phonon coupling effects for the migration enthalpy calculation as the barrier energy for vacancy migration is calculated more accurately according to zero-temperature energies compared to the bar- 
rier energy for vacancy formation. This is because two more similar structures that both contain monovacancies are compared. Brute-force AIMD simulations cannot be used to calculate the enthalpy of the intermediate state since the simulation quickly slips away from this out-of-equilibrium state. A more sophisticated sampling technique is required which is the subject of a current development by the authors. The vacancy formation enthalpy is the difference of enthalpies for the bulk and defected systems and is obtained from $a b$ initio molecular dynamics (AIMD) average energy and pressure (see section SII in [51] for more details). Due to underestimation of DFT in obtaining the energy of the intrinsic surface formed around a vacancy, we add an explicit correction term to both the migration and vacancy formation enthalpies according to the electron density of the exchange-correlation functional [68, 69] (see section SIII in [51] for more details). Fig. 4(a) illustrates the vacancy formation enthalpy for bcc titanium above the allotropic transformation temperature (a similar plot is presented for bcc zirconium in Fig. S9(d) in Supplemental Materials [51]).

Vacancy formation entropy is obtained by comparing the temperature-dependent phonon frequencies and DOS for the bulk and defected systems. Fig. 3(a)-(b) illustrates the temperature-dependent phonon dispersion and DOS for both systems at $1600 \mathrm{~K}$ obtained using the TDEP method [63, 64] (similar plots for bcc Zr are shown in Fig. S7 in Supplemental Materials [51]). The formation of a vacancy results in appearance of optical phonon branches by removing the periodicity of the crystal structure and it broadens the peak in the DOS due to existence of several localized modes at higher frequencies. The vibration entropy for the bulk and defected systems are obtained based on T-dependent phonon frequencies, $\omega(T)$, and DOS, $g(\omega(T))$ (see section SIV in Supplemental Materials [51]). Fig.3(c)-(d) shows the anharmonic phonon density of states for both the bulk phase and the bcc phase including a monovacancy obtained by using the TDEP method at different temperatures (similar plots for bcc Zr are shown in Fig. S10 in Supplemental Materials [51]). Creating a vacancy in the supercell results in larger number of internal degrees of freedom, in this case $216 \times 3-3=645$, which is the area under the phonon density of states. The vacancy formation entropy for each temperature is shown in Fig. 4(b). As illustrated in Fig. 4(c), we calculate the equilibrium monovacancy concentration, $C_{v}$, having the formation entropy $\Delta S_{v}$ and formation enthalpy $\Delta H_{v}$ (see section SIV in Supplemental Materials [51]).

The $a b$ initio predicted diffusivity for bcc titanium and zirconium alongside the parameters used in Eq. 2 are presented in Fig. 5. The circled-curve in Fig. 5 are the diffusivity predictions by including the anharmonic lattice vibration effects, which agrees well with experimental measurements of Ref. [40]. The diffusivity prediction based on the harmonic phonon analysis underestimates the diffusion coefficient by several orders of magnitude as shown by the crossed curve in Fig. 5 . In spite of the fact that we use identical migration and formation enthalpies in both approaches, namely the proposed approach based on anharmonic phonon analysis and the standard harmonic model, the calculated diffusivities differ dramatically. Moreover, the vacancy formation entropy and concentration according to the proposed approach and the harmonic model are very similar for both bcc $\mathrm{Ti}$ and $\mathrm{Zr}$ (see Fig. 4(b)-(c) and Fig. S11 in Supplemental Materials [51]). This further illustrates the significant role of anharmonic lattice vibrations in enhancing diffusivity in the bcc phase of $\mathrm{Ti}$ and $\mathrm{Zr}$. The harmonic lattice vibration model underestimates the diffusivity by almost 3 orders of magnitude and 2 orders of magnitude for bcc $\mathrm{Ti}$ and bcc Zr, respectively (see next section for more details on harmonic model prediction).

\section{SIGNIFICANCE OF PHONON-PHONON COUPLING EFFECTS}

To understand the significance of anharmonic vibration effects, we also calculated the diffusion coefficient according to a standard harmonic phonon analysis by excluding the imaginary harmonic phonon frequencies to calculate the vacancy formation entropy (see section SIV in [51]). We also use the T-independent value $\nu_{1} \times \frac{\prod_{i} \nu_{i}}{\prod_{i} \nu_{i}^{\prime}}$ to obtain the $\nu^{*}$ in Eq. 2 by assuming imaginary $\nu$ and $\nu^{\prime}$ frequencies have real values. $\nu_{1}$ is the most unstable phonon frequency (i.e., the square root of the most negative eigenvalue of the dynamical matrix) for the transition state. The transition state is assumed to be the estimated intermediate state presented in Fig. 1(d) for bcc Ti and in Fig. S5(d) for bcc Zr. We calculate $\nu_{1}$ to be $6.64 \mathrm{THz}$ and $4.63 \mathrm{THz}$ for $\mathrm{Ti}$ and $\mathrm{Zr}$, respectively. This value is comparable to the temperature-dependent $\nu^{*}$ in our approach, presented in Fig. 5. However, the effective jump prefactor $\nu^{*}$ is severely low in the harmonic approximation. This is due to the small value for $\frac{\prod_{i} \nu_{i}}{\prod_{i} \nu_{i}^{\prime}}$, which essentially assumes phonon modes are independent (decoupled) oscillators and neglect phonon-phonon coupling effects. This assumption is severely underperforming for calculating diffusion coefficient in phases which harmonic phonon instabilities not simply due to infeasibility of its application but more importantly because of the dominant effect of anharmonic phonon interactions. We calculate the ratio of the product of independent harmonic phonon frequencies in the initial and intermediate states, $\frac{\prod_{i} \nu_{i}}{\prod_{i} \nu_{i}^{\prime}}$, to be 0.0011 and 0.0073 for bcc $\mathrm{Ti}$ and $\mathrm{Zr}$, respectively, which results in $\nu^{*}$ values of $0.0073 \mathrm{THz}$ and $0.034 \mathrm{THz}$ for bcc $\mathrm{Ti}$ and $\mathrm{Zr}$, respectively. Typical ratio values of $\frac{\prod_{i} \nu_{i}}{\prod_{i} \nu_{i}^{\prime}}$ for solid phases that are mechanically stable and can be accurately described by harmonic phonon modes are orders of magnitude higher. For example, the ratio value for fcc $\mathrm{Al}$ is 5.6 and for fcc $\mathrm{Ag}$ is 6.94. We obtained these values by comparing the DFT harmonic phonon eigenvalues for the initial and saddle point con- 
a)

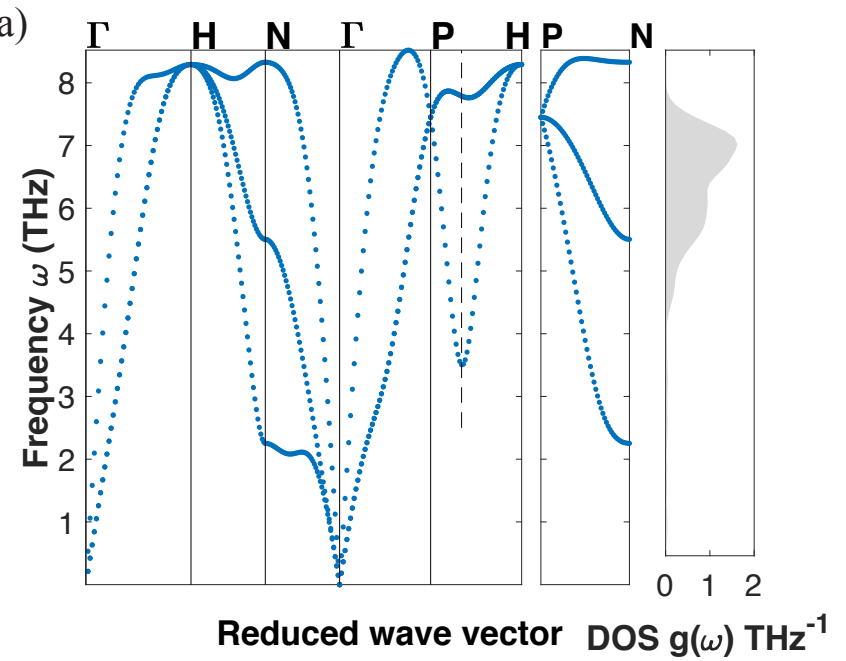

c)

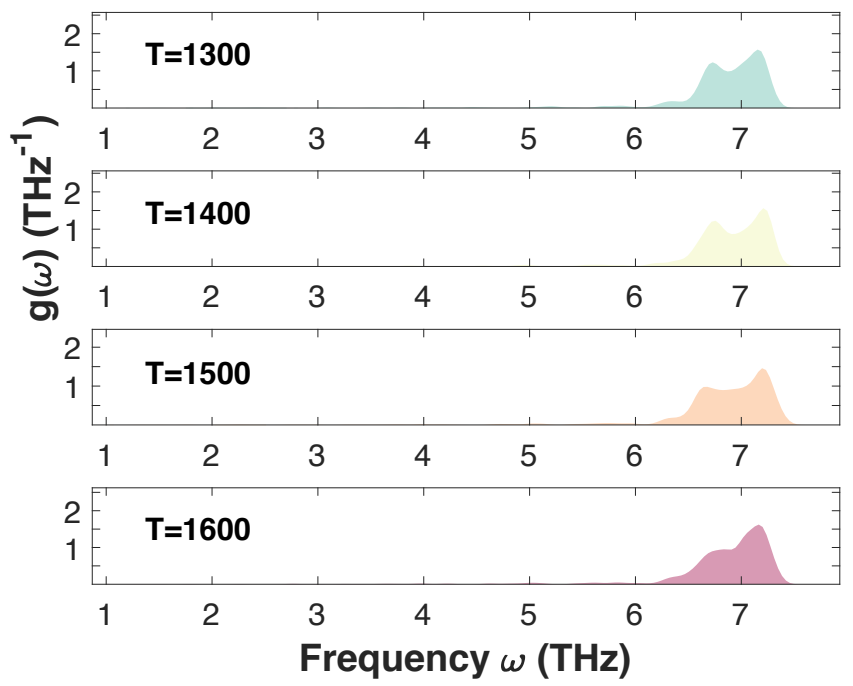

b)

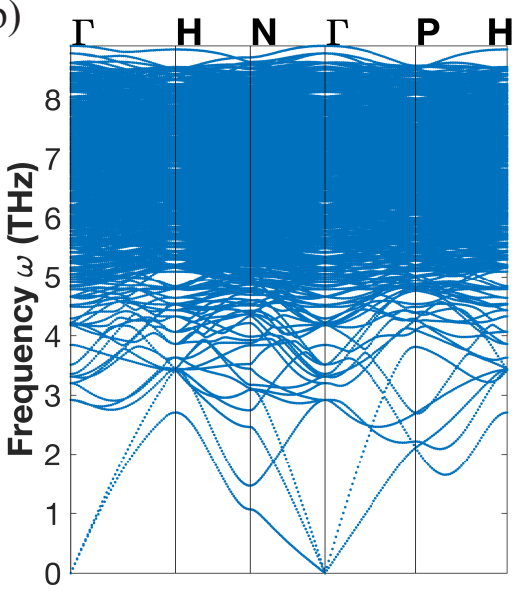

$P \quad N$

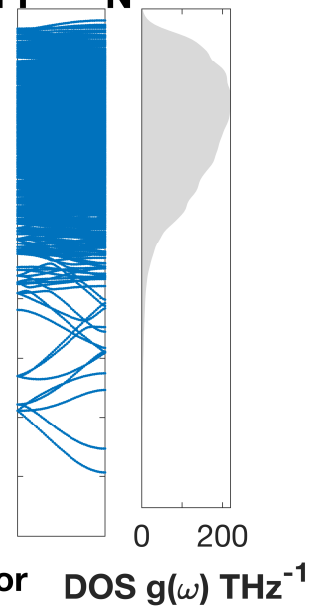

d)

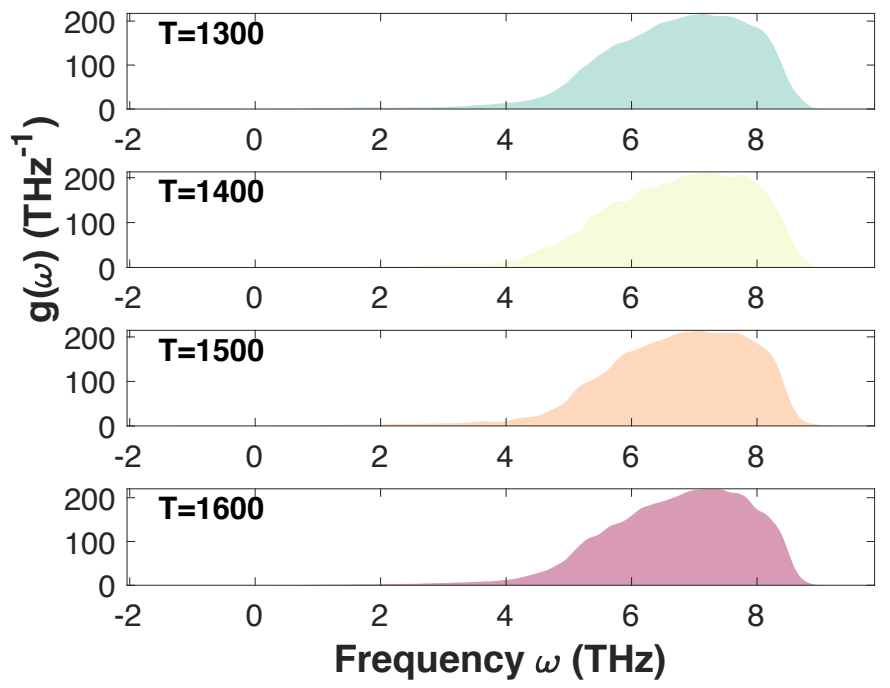

FIG. 3. The anharmonic phonon dispersion and density of states (DOS) for a) a $6 \times 6 \times 6$ supercell of bcc titanium including 216 atoms and b) bcc Ti with a monovacancy including 215 atoms at $1600 \mathrm{~K}$. c) The temperature-dependent DOS for bulk bcc $\mathrm{Ti}$ and d) the bcc phase with a monovacany at different temperatures.

figuration obtained from the nudged elastic band method and are reported in our previous work [50].

\section{COMPUTATIONAL DETAILS}

\section{A. DFT calculation}

Electronic structure calculations are performed using density functional theory (DFT) as implemented in the Vienna Ab-initio Simulation Package (VASP) [7073]. We use the projector augmented wave (PAW) method [74,75] with energy cutoff of $274.6 \mathrm{eV}$ for $\mathrm{Ti}$ and $229.9 \mathrm{eV}$ for $\mathrm{Zr}$ and the generalized gradient approximation (GGA) [72, 73, 76] with the PBE [77] exchangecorrelation (Ti_pv and Zr_sv). All DFT calculations, in- cluding static energy, molecular dynamics and SCAILD are performed on a $6 \times 6 \times 6$ supercell of bcc including 216 atoms and a supercell with a vacancy at the bcc site. For bcc Cr, we use the energy cutoff of $395.5 \mathrm{eV}$ for the PAW psuedopotential with the PBE [77] exchange-correlation functional for a supercell of 128 atoms for the bulk phase and 127 atoms for the bcc phase with a monovacancy.

\section{B. Enthalpy calculations}

AIMD simulations are performed at constant volume and temperature (NVT) under the Nose-Hoover thermostat [78-82]. Enthalpy values for the bulk and defected systems are obtained by averaging AIMD kinetic and potential energies and the pressure-volume term that 


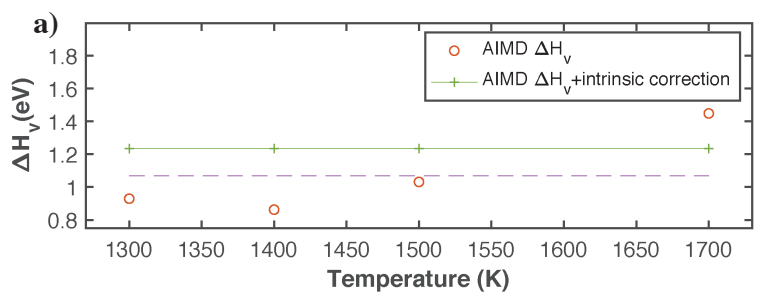

b)

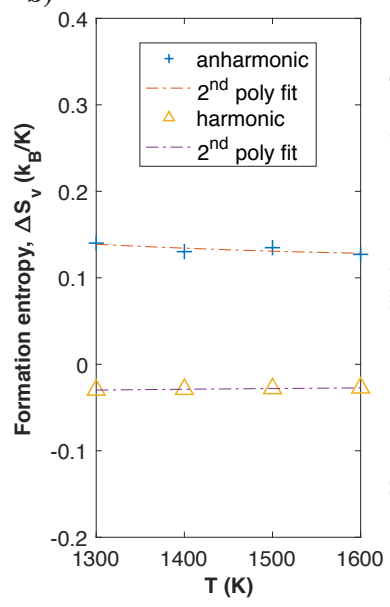

c)

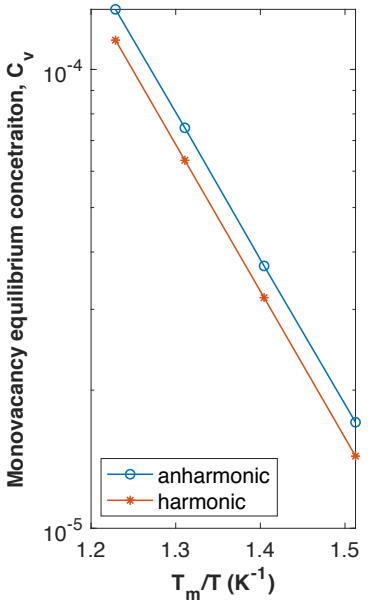

FIG. 4. a) The vacancy formation enthalpy in bcc Ti as a function of temperature. The circles are the statical average formation enthalpy obtained from AIMD simulation using the PBE exchange-correlation functional. The crosses are the formation enthalpy values including the explicit intrinsic surface correction term. b) The formation entropy and c) equilibrium vacancy concentration versus temperature for monovacany in bcc Ti. For bulk bcc we used a $6 \times 6 \times 6$ supercell of bcc titanium including 216 atoms and for defected system we used bcc Ti with a monovacancy including 215 atoms.

are captured every $0.1 \mathrm{ps}$ for a 35 ps trajectory after 1 ps thermalization at each temperature (see section SII in [51] for more details).

\section{Phonon analysis}

SCAILD iteratively converges the phonon frequencies at different temperatures accounting for anharmonic effects [60-62]. In TDEP, temperature-dependent forceconstant tensor is obtained by minimizing the difference between $a b$ initio molecular dynamics (AIMD) forces and forces described in the harmonic approximation [63, 64] (see section SI in [51] for details). SCAILD method is performed using the SCPH code [61] based on DFT calculations until the free energy difference converges to $10^{-5} \mathrm{eV}$ (see section SI in [51]). For the DFT calculations the Brillouin zone integration is performed on a mesh density of 4000 points per reciprocal angstrom (generated by the ezvasp module [83]). In TDEP method, phonon dispersions and DOSs are obtained from temperature-dependent force constant tensors using the Phonopy code [84]. The temperature-dependent force constant tensor is obtained by least square fitting of AIMD forces and displacements to a harmonic model using our in-house code. For AIMD simulations, the first Brillouin zone integration is performed on the $\Gamma$ point for a $6 \times 6 \times 6$ supercell of bcc with cell volume of 3799.45 $\AA^{3}$ and $4988.63 \AA^{3}$ for $\mathrm{Ti}$ and $\mathrm{Zr}$, respectively.

\section{DISCUSSION}

The agreement of our first-principles prediction with experimental diffusion coefficient indicates that monovacancy jumps are the dominant atomic mechanism responsible for macroscopic diffusion in the bcc phase of IIIB-IVB metals. The anomalously larger diffusivity in bcc IIIB-IVB metals compared to other bcc and fcc metals can be accurately accounted for by including temperature-dependent anharmonic lattice vibration effects within the transition state theory to describe monvacancy jumps. In other words, monovacancy jumps are significantly promoted due to phonon-phonon coupling effects in these phases. On the other hand, the nature of free energy surface in bcc IIIB-IVB metals, which consists of multiple local minima around the bcc structure, results in hopping of the system among local structural distortions (this has been shown for bcc Ti and B2 NiTi in our previous studies $[22,28]$ ) and increases the likelihood of collective atomic motion. This phenomenon has been observed as heterophase fluctuations in earlier works [41, 85] and as a concerted string-like atomic motion in a more recent ab initio molecular dynamics simulation [44]. However, our calculations shows that these collective motions do not contribute to the macroscopic diffusivity, in other words they do not result in a long range mass transport. The contribution of collective atomic motion to diffusivity can be the subject of a future investigation which requires long-time molecular dynamics simulation (which are feasible based on potential models) for a complete statistical sampling of the diffusion process (similar to methods used to distinguish cyclic collective motion in tetragonal Li7La3Zr2O12 (LLZO) [86]). One possibility is that these collective atomic motions are cyclic or has a random nature with a zero long-range effect. Unfortunately, the current potential models for bcc $\mathrm{Ti}$ (e.g., the MEAM potential [87]) cannot effectively capture the softening of $L \frac{2}{3}\langle 111\rangle$ phonon, which plays the major role in softening of restoring forces and collective atomic distortions. The phonon-assisted vacancy jump mechanism to be the predominant one in bcc IVB metals is supported by direct experimental determination of the mechanism [31-33, 35] and the isotope effect [46, 47].

While self-consistent harmonic approximation (SCHA) approaches (e.g., SCAILD and TDEP) have been extensively used for thermodynamic description of various systems [61-64, 88-91], we utilize self-consistent temperature-dependent phonon analysis to describe dif- 


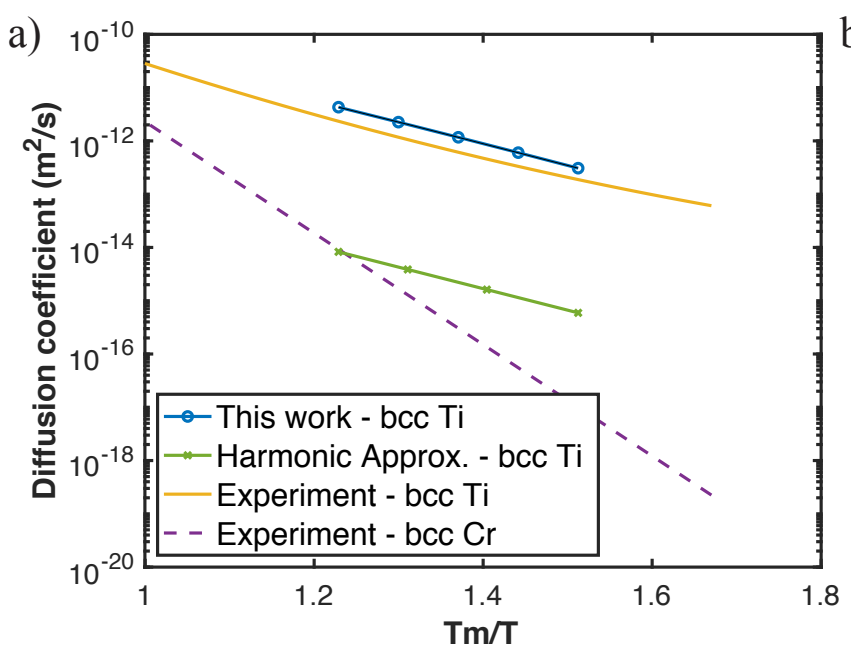

\begin{tabular}{|l|l|l|l|l|}
\hline \multicolumn{5}{|c|}{ temperature-dependent values } \\
\hline $\mathrm{T}(\mathrm{K})$ & 1300 & 1400 & 1500 & 1600 \\
\hline $\mathrm{v}^{*}(\mathrm{THz})$ & 3.211 & 3.280 & 3.291 & 3.289 \\
\hline$\Delta \mathrm{S}_{\mathrm{v}}\left(\mathrm{k}_{\mathrm{b}} / \mathrm{K}\right)$ & 0.140 & 0.130 & 0.135 & 0.127 \\
\hline \multicolumn{5}{|c|}{ temperature-independent values } \\
\hline$\Delta \mathrm{H}_{\mathrm{m}}=0.274+(0.053) \mathrm{eV}$ & $\Delta \mathrm{H}_{\mathrm{v}}=1.06+(0.182) \mathrm{eV}$ \\
\hline$\Delta \mathrm{S}_{\mathrm{m}} \approx 0$ & $\alpha=8 / 6$ & $\mathrm{a}=1 / 2 \mathrm{a}_{0}[111]=\sqrt{3} / 2 \mathrm{a}_{0} \AA$ & $\mathrm{a}_{0}=3.28 \AA$ \\
\hline
\end{tabular}

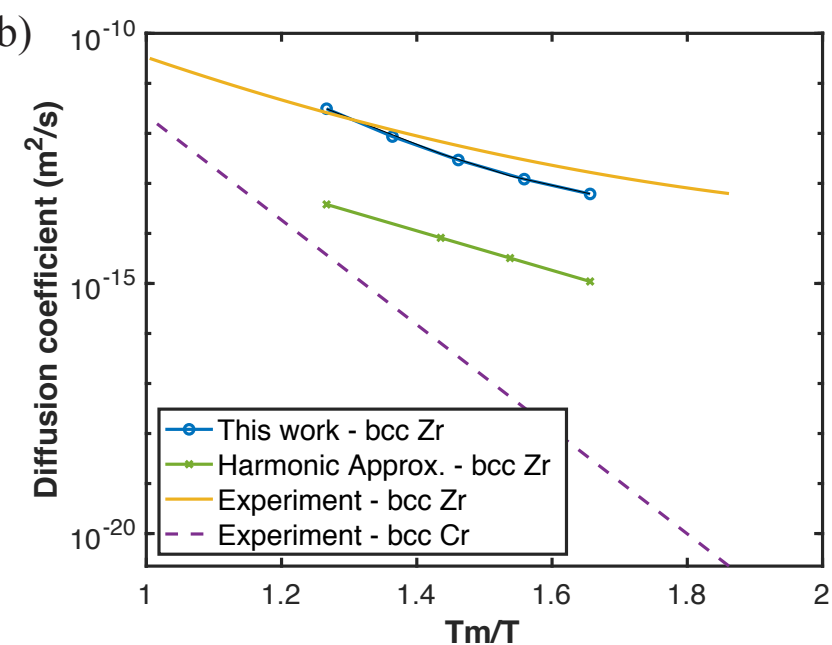

\begin{tabular}{|l|c|c|c|c|}
\hline \multicolumn{5}{|c|}{ temperature-dependent values } \\
\hline $\mathrm{T}(\mathrm{K})$ & 1300 & 1400 & 1500 & 1700 \\
\hline$v^{*}(\mathrm{THz})$ & 2.11 & 2.09 & 2.11 & 2.12 \\
\hline$\Delta \mathrm{S}_{\mathrm{v}}\left(\mathrm{k}_{\mathrm{b}} / \mathrm{K}\right)$ & -0.23 & -0.48 & -0.4 & 0.132 \\
\hline \multicolumn{5}{|c|}{ temperature-independent values } \\
\hline$\Delta \mathrm{H}_{\mathrm{m}}=0.403+(0.049) \mathrm{eV}$ & $\Delta \mathrm{H}_{\mathrm{v}}=1.068+(0.165) \mathrm{eV}$ \\
\hline$\Delta \mathrm{S}_{\mathrm{m}} \approx 0$ & $\alpha=8 / 6$ & $\mathrm{a}=1 / 2 \mathrm{a}_{0}[111]=\sqrt{ } 3 / 2 \mathrm{a}_{0} \AA$ & $\mathrm{a}_{0}=3.59 \AA$ \\
\hline
\end{tabular}

FIG. 5. Complete DFT-based prediction of diffusion coefficient for a) bcc Ti and b) bcc Zr obtained according to anharmonic phonon analysis (circled-curves), validated with experimental measurements (solid curve) extracted from Ref. [40]. Diffusion coefficient for bcc Cr (dashed curve) is also extracted from Ref. [40] for comparison. Calculations based on standard harmonic phonon analysis (crosses) underestimates the diffusion coefficient by several orders of magnitude. $T_{m}$ is equal to $1966 \mathrm{~K}$ and $2153 \mathrm{~K}$ for Ti and Zr, respectively. All the parameters for calculating the diffusion coefficient are presented in the tables. The internal surface correction terms for vacancy formation and migration enthalpies are presented in parentheses. $\nu^{*}$ for each temperature is also represented by star marks in Fig. 2(d) for bcc Ti and Fig. S6 for bcc Zr.

fusive jump rates within the framework of the transition state theory for the first time to the best of our knowledge. The introduced approach can be used for diffusion description of other mechanically unstable phases, including the $\beta$ phase of IIIB and IVB metals and their alloys. The computation cost of the introduced methodology is minimal, only requiring anharmonic phonon analysis of two systems (a bulk phase and a small supercell including a vacancy) at each temperature, when compared to diffusion description based on brute-force molecular dynamic simulation in the order of nano seconds (refer to Ref. [86] for details on adequate MD sampling of diffusive events).

Developing a general framework that effectively accounts for temperature-induced effects in diffusion description of mechanically unstable but dynamically stabilized phases is beyond the scope of this report and is the subject of an active investigation by the authors. The major assumption in calculation of diffusivity in this re- port is the approximation of migration energy barrier and the effective jump frequency along the migration path (details are described in section III). These assumption are justified for the pure bcc phase due to the coincidence of diffusive jump direction and a specific phonon mode with softening effects.

In summary, we predict the diffusivity of the bcc Ti and Zr by simply including temperature-induced anharmonic phonon-phonon coupling effects within the monovacancymediated diffusive jump process. Our predictions are in good agreement with experimental measurements while disregarding the anharmonic vibration effects results in orders of magnitude underestimation in diffusivity. Our findings shed light on the underlying mechanism responsible for markedly larger diffusion coefficient in bcc IIIB and IVB metals and provide a computationally efficient and accurate approach for macroscopic diffusivity calculations beyond the harmonic lattice vibration approximation. 


\section{ACKNOWLEDGMENTS}

We would like to thank Dr. John W. Lawson and Dr. Justin Haskins from NASA Ames Research Center for kindly sharing their code for T-dependent force-constant tensor calculation. Our in-house code is a modification of their code. Some of the calculations in this work were performed on the Extreme Science and Engineering Discovery Environment (XSEDE) resource through allocation TG-DMR190017 [92].

[1] M. Mantina, Y. Wang, R. Arroyave, L. Q. Chen, Z. K. Liu, and C. Wolverton, "First-Principles Calculation of Self-Diffusion Coefficients," Physical Review Letters 100, 215901 (2008).

[2] M. Mantina, Y. Wang, L.Q. Chen, Z.K. Liu, and C. Wolverton, "First principles impurity diffusion coefficients," Acta Materialia 57, 4102-4108 (2009).

[3] Henry H. Wu and Dallas R. Trinkle, "Direct diffusion through interpenetrating networks: Oxygen in titanium," Phys. Rev. Lett. 107, 045504 (2011).

[4] Erich Wimmer, Walter Wolf, Jürgen Sticht, Paul Saxe, Clint B. Geller, Reza Najafabadi, and George A. Young, "Temperature-dependent diffusion coefficients from ab initio computations: Hydrogen, deuterium, and tritium in nickel," Phys. Rev. B 77, 134305 (2008).

[5] Marina V. Koudriachova, Nicholas M. Harrison, and Simon W. de Leeuw, "Diffusion of Li-ions in rutile. An ab initio study," Solid State Ionics 157, 35 - 38 (2003), proceedings of the 6th International Symposium on Systems with Fast Ionic Transport (ISSFIT).

[6] C. Domain and C. S. Becquart, "Diffusion of phosphorus in $\alpha$-Fe: An ab initio study," Phys. Rev. B 71, 214109 (2005).

[7] J.D. Tucker, R. Najafabadi, T.R. Allen, and D. Morgan, "Ab initio-based diffusion theory and tracer diffusion in NiCr and NiFe alloys," Journal of Nuclear Materials 405, $216-234$ (2010).

[8] J. Sarnthein, K. Schwarz, and P. E. Blöchl, "Ab initio molecular-dynamics study of diffusion and defects in solid $\mathrm{Li}_{3} \mathrm{~N}, "$ Phys. Rev. B 53, 9084-9091 (1996).

[9] S. Choudhury, L. Barnard, J.D. Tucker, T.R. Allen, B.D. Wirth, M. Asta, and D. Morgan, "Ab-initio based modeling of diffusion in dilute bcc FeNi and FeCr alloys and implications for radiation induced segregation," Journal of Nuclear Materials 411, 1 - 14 (2011).

[10] Peter E. Blöchl, C. G. Van de Walle, and S. T. Pantelides, "First-principles calculations of diffusion coefficients: Hydrogen in silicon," Phys. Rev. Lett. 64, 14011404 (1990).

[11] Anton Van der Ven, John C. Thomas, Qingchuan Xu, Benjamin Swoboda, and Dane Morgan, "Nondilute diffusion from first principles: Li diffusion in $\mathrm{Li}_{x} \mathrm{TiS}_{2}$," Phys. Rev. B 78, 104306 (2008).

[12] A. Van der Ven, G. Ceder, M. Asta, and P. D. Tepesch, "First-principles theory of ionic diffusion with nondilute carriers," Phys. Rev. B 64, 184307 (2001).

[13] Mikhail I. Mendelev and Yuri Mishin, "Molecular dynamics study of self-diffusion in bcc Fe," Physical Review B 80, 144111 (2009).

[14] V. Milman, M. C. Payne, V. Heine, R. J. Needs, J. S. Lin, and M. H. Lee, "Free energy and entropy of diffusion by ab initio molecular dynamics: Alkali ions in silicon," Physical Review Letters 70, 2928-2931 (1993).
[15] W. Frank, U. Breier, C. Elsässer, and M. Fähnle, "FirstPrinciples Calculations of Absolute Concentrations and Self-Diffusion Constants of Vacancies in Lithium," Physical Review Letters 77, 518-521 (1996).

[16] Henry Eyring, "The activated complex in chemical reactions," The Journal of Chemical Physics 3, 107-115 (1935), https://doi.org/10.1063/1.1749604.

[17] George H. Vineyard, "Frequency factors and isotope effects in solid state rate processes," Journal of Physics and Chemistry of Solids 3, 121-127 (1957).

[18] Göran Grimvall, Blanka Magyari-Köpe, Vidvuds Ozolinš, and Kristin A. Persson, "Lattice instabilities in metallic elements," Reviews of Modern Physics 84, 945-986 (2012).

[19] K. Einarsdotter, B. Sadigh, G. Grimvall, and V. Ozolinš, "Phonon Instabilities in fcc and bcc Tungsten," Physical Review Letters 79, 2073-2076 (1997).

[20] A. P. Mirgorodsky, M. B. Smirnov, and P. E. Quintard, "Lattice-dynamical study of the cubic-tetragonalmonoclinic transformations of zirconia," Physical Review B 55, 19-22 (1997).

[21] K. Parlinski, Z. Q. Li, and Y. Kawazoe, "First-Principles Determination of the Soft Mode in Cubic ZrO 2," Physical Review Letters 78, 4063-4066 (1997).

[22] Sara Kadkhodaei, Qi-Jun Hong, and Axel van de Walle, "Free energy calculation of mechanically unstable but dynamically stabilized bcc titanium," Physical Review B 95, 064101 (2017).

[23] Kristin Persson, Mathias Ekman, and Vidvuds Ozolinš, "Phonon instabilities in bcc Sc, Ti, La, and Hf," Physical Review B 61, 11221-11224 (2000).

[24] P. J. Craievich, J. M. Sanchez, R. E. Watson, and M. Weinert, "Structural instabilities of excited phases," Physical Review B 55, 787-797 (1997).

[25] Y. Y. Ye, Y. Chen, K. M. Ho, B. N. Harmon, and P. A. Lindgard, "Phonon-phonon coupling and the stability of the high-temperature bcc phase of Zr," Physical Review Letters 58, 1769-1772 (1987).

[26] Qingchuan Xu and Anton Van der Ven, "First-principles investigation of metal-hydride phase stability: The Ti-H system," Physical Review B 76, 064207 (2007).

[27] Ramiro Quijano, Romeo de Coss, and David J. Singh, "Electronic structure and energetics of the tetragonal distortion for $\mathrm{TiH} 2, \mathrm{ZrH} 2$, and $\mathrm{HfH} 2$ : A first-principles study," Physical Review B 80, 184103 (2009).

[28] Sara Kadkhodaei and Axel van de Walle, "Firstprinciples calculations of thermal properties of the mechanically unstable phases of the PtTi and NiTi shape memory alloys," Acta Materialia 147, 296 - 303 (2018).

[29] A. T. Zayak, P. Entel, J. Enkovaara, A. Ayuela, and R. M. Nieminen, "First-principles investigation of phonon softenings and lattice instabilities in the shapememory system Ni 2 MnGa," Physical Review B 68, 
$132402(2003)$

[30] G. Vogl, W. Miekeley, A. Heidemann, and W. Petry, "Anomalously fast diffusion of cobalt in $\beta$-zirconium: Evidence for two different jump frequencies from quasielastic neutron scattering," Phys. Rev. Lett. 53, 934-937 (1984).

[31] G. Vogl, W. Petry, Th Flottmann, and A. Heiming, "Direct determination of the self-diffusion mechanism in bcctitanium," Physical Review B 39, 5025-5034 (1989).

[32] R.F Peart and D.H Tomlin, "Diffusion of solute elements in beta-titanium," Acta Metallurgica 10, 123 134 (1962).

[33] Winfried Petry, A. Heiming, J. Trampenau, and G. Vogl, "On the Diffusion Mechanism in the bcc Phase of the Group 4 Metals," in Diffusion in Metals and Alloys, Defect and Diffusion Forum, Vol. 66 (Trans Tech Publications, 1991) pp. 157-174.

[34] Christian Herzig, J. Neuhaus, K. Vieregge, and L. Manke, "Fast Impurity Diffusion of Co and Fe in $\beta-\mathrm{Zr}$ and $\beta$-Zr-Alloys," in Vacancies and Interstitials in Metals and Alloys, Materials Science Forum, Vol. 15 (Trans Tech Publications, 1987) pp. 481-486.

[35] W. Petry, T. Flottmann, A. Heiming, J. Trampenau, M. Alba, and G. Vogl, "Atomistic study of anomalous self-diffusion in bcc $\beta$-titanium," Phys. Rev. Lett. 61, 722-725 (1988).

[36] Chr. Herzig, U. Khler, and S. V. Divinski, "Tracer diffusion and mechanism of non-Arrhenius diffusion behavior of $\mathrm{Zr}$ and $\mathrm{Nb}$ in body-centered cubic $\mathrm{ZrNb}$ alloys," Journal of Applied Physics 85, 8119-8130 (1999), https://doi.org/10.1063/1.370650.

[37] H. M. Gilder and D. Lazarus, "Role of vacancy anharmonicity on non-Arrhenius diffusional behavior," Phys. Rev. B 11, 4916-4926 (1975).

[38] J. M. Sanchez and D. De Fontaine, "Model for anomalous self-diffusion in group-IVB transition metals," Physical Review Letters 35, 227-230 (1975).

[39] J.M. Sanchez and D. De Fontaine, "Anomalous diffusion in omega forming systems," Acta Metallurgica 26, 1083 - 1095 (1978).

[40] Christian Herzig and U. Köhler, "Anomalous SelfDiffusion in BCC IVB Metals and Alloys," Materials Science Forum 15-18, 301-322 (1987).

[41] G Vogl, "Fast solute diffusion in metals and the tendency for heterophase fluctuations," Europhysics Letters (EPL) 13, 149-154 (1990).

[42] Chr. Herzig, "The correlation between diffusion behaviour and phonon softening in bcc metals," Berichte der Bunsengesellschaft fr physikalische Chemie 93, 12471252 (1989).

[43] Sergei L. Dudarev, "The non-arrhenius migration of interstitial defects in bcc transition metals," Comptes Rendus Physique 9, 409 - 417 (2008), materials subjected to fast neutron irradiation.

[44] D. G. Sangiovanni, J. Klarbring, D. Smirnova, N. V. Skripnyak, D. Gambino, M. Mrovec, S. I. Simak, and I. A. Abrikosov, "Superioniclike diffusion in an elemental crystal: bcc titanium," Phys. Rev. Lett. 123, 105501 (2019).

[45] Mikhail I. Mendelev and Boris S. Bokstein, "Molecular dynamics study of self-diffusion in zr," Philosophical Magazine 90, 637-654 (2010), https://doi.org/10.1080/14786430903219020.
[46] L. Manke and C. Herzig, "Diffusion und isotopieeffekt von silber in $\beta$-Zirkon," Acta Metallurgica 30, 2085-2092 (1982).

[47] Mark S. Jackson and David Lazarus, "Isotope effect for diffusion of tin in $\beta$-titanium," Physical Review B 15, 4644-4656 (1977).

[48] Paul Shewmon, Diffusion in Solids (Springer International Publishing, 2016).

[49] Helmut Mehrer, Diffusion in Solids: Fundamentals, Methods, Materials, Diffusion-Controlled Processes (Springer, Berlin, Heidelberg, 2007).

[50] S. Kadkhodaei and A. van de Walle, "A simple local expression for the prefactor in transition state theory," The Journal of Chemical Physics 150, 144105 (2019), https://doi.org/10.1063/1.5086746.

[51] See Supplemental Material at for details on calculation of anharmonic phonon dispersion and density of states, vacancy formation enthalpy, vacancy internal surface energy correction term, vacancy formation entropy and equilibrium concentration, and details for diffusivity calculation in bcc zirconium.

[52] Sara Kadkhodaei and Axel van de Walle, "Software tools for thermodynamic calculation of mechanically unstable phases from first-principles data," Computer Physics Communications 246, 106712 (2020).

[53] V. Ozolins, "First-Principles Calculations of Free Energies of Unstable Phases: The Case of fcc W," Physical Review Letters 102, 065702 (2009).

[54] John C. Thomas and Anton Van der Ven, "Elastic properties and stress-temperature phase diagrams of hightemperature phases with low-temperature lattice instabilities," Physical Review B 90, 224105 (2014).

[55] R. G. Hennig, T. J. Lenosky, D. R. Trinkle, S. P. Rudin, and J. W. Wilkins, "Classical potential describes martensitic phase transformations between the $\alpha, \beta$, and $\omega$ titanium phases," Physical Review B 78, 054121 (2008).

[56] Zhi-Gang Mei, Shun-Li Shang, Yi Wang, and Zi-Kui Liu, "Density-functional study of the thermodynamic properties and the pressuretemperature phase diagram of $\mathrm{Ti}$," Physical Review B 80, 104116 (2009).

[57] Fei Zhou, Weston Nielson, Yi Xia, and Vidvuds Ozolinš, "Lattice Anharmonicity and Thermal Conductivity from Compressive Sensing of First-Principles Calculations," Physical Review Letters 113, 185501 (2014).

[58] Andrew Ian Duff, Theresa Davey, Dominique Korbmacher, Albert Glensk, Blazej Grabowski, Jörg Neugebauer, and Michael W. Finnis, "Improved method of calculating ab initio high-temperature thermodynamic properties with application to ZrC," Physical Review B 91, 214311 (2015).

[59] Jefferson Z. Liu, A. van de Walle, G. Ghosh, and M. Asta, "Structure, energetics, and mechanical stability of fe-cu bcc alloys from first-principles calculations," Phys. Rev. B 72, 144109 (2005).

[60] N. R. Werthamer, "Self-Consistent Phonon Formulation of Anharmonic Lattice Dynamics," Physical Review B 1, 572-581 (1970).

[61] P. Souvatzis, O. Eriksson, M. I. Katsnelson, and S. P. Rudin, "Entropy Driven Stabilization of Energetically Unstable Crystal Structures Explained from First Principles Theory," Physical Review Letters 100, 095901 (2008).

[62] P. Souvatzis, O. Eriksson, M.I. Katsnelson, and S.P. Rudin, "The self-consistent ab initio lattice dynamical 
method," Computational Materials Science 44, 888- 894 (2009).

[63] O. Hellman, I. A. Abrikosov, and S. I. Simak, "Lattice dynamics of anharmonic solids from first principles," Physical Review B 84, 180301(R) (2011).

[64] Olle Hellman, Peter Steneteg, I. A. Abrikosov, and S. I. Simak, "Temperature dependent effective potential method for accurate free energy calculations of solids," Physical Review B 87, 104111 (2013).

[65] Graeme Henkelman and Hannes Jónsson, "Improved tangent estimate in the nudged elastic band method for finding minimum energy paths and saddle points," The Journal of Chemical Physics 113, 9978-9985 (2000).

[66] Graeme Henkelman, Blas P. Uberuaga, and Hannes Jónsson, "A climbing image nudged elastic band method for finding saddle points and minimum energy paths," The Journal of Chemical Physics 113, 9901-9904 (2000).

[67] Graeme Henkelman and Hannes Jónsson, "A dimer method for finding saddle points on high dimensional potential surfaces using only first derivatives," The Journal of Chemical Physics 111, 7010-7022 (1999).

[68] Karin Carling, Göran Wahnström, Thomas R. Mattsson, Ann E. Mattsson, Nils Sandberg, and Göran Grimvall, "Vacancies in metals: From first-principles calculations to experimental data," Phys. Rev. Lett. 85, 3862-3865 (2000).

[69] Thomas R. Mattsson and Ann E. Mattsson, "Calculating the vacancy formation energy in metals: $\mathrm{Pt}, \mathrm{pd}$, and mo," Phys. Rev. B 66, 214110 (2002).

[70] G. Kresse and J. Hafner, "Ab initio molecular dynamics for liquid metals," Phys. Rev. B 47, 558-561 (1993).

[71] G. Kresse and J. Furthmller, "Efficiency of ab-initio total energy calculations for metals and semiconductors using a plane-wave basis set," Computational Materials Science 6, 15 - 50 (1996).

[72] G. Kresse and J. Furthmüller, "Efficient iterative schemes for ab initio total-energy calculations using a plane-wave basis set," Phys. Rev. B 54, 11169-11186 (1996).

[73] G. Kresse and D. Joubert, "From ultrasoft pseudopotentials to the projector augmented-wave method," Phys. Rev. B 59, 1758-1775 (1999).

[74] P. E. Blöchl, "Projector augmented-wave method," Phys. Rev. B 50, 17953-17979 (1994).

[75] G. Kresse and D Joubert, "From ultrasoft pseudopotentials to the projector augmented-wave method." Phys. Rev. B 59, 1758-1775 (1999).

[76] John P. Perdew, "Generalized gradient approximation for the fermion kinetic energy as a functional of the density," Physics Letters A 165, 79 - 82 (1992).

[77] J. P. Perdew, K. Burke, and M. Ernzerhof, "Generalized gradient approximation made simple." Phys. Rev. Lett. 77, 3865-3868 (1996).

[78] William G. Hoover and Brad Lee Holian, "Kinetic moments method for the canonical ensemble distribution," Physics Letters A 211, 253 - 257 (1996).

[79] S. Nosé, "A molecular dynamics method for simulations in the canonical ensemble," Molecular Physics 52, 255268 (1984).

[80] Shuichi Nosé, "A unified formulation of the constant temperature molecular dynamics methods," The Journal of Chemical Physics 81, 511-519 (1984).

[81] William G. Hoover, "Canonical dynamics: Equilibrium phase-space distributions," Phys. Rev. A 31, 1695 (1985).
[82] Glenn J. Martyna, Michael L. Klein, and Mark Tuckerman, "Nosé-Hoover chains: The canonical ensemble via continuous dynamics," The Journal of Chemical Physics 97, 2635-2643 (1992).

[83] A. van de Walle and G. Ceder, "Automating firstprinciples phase diagram calculations," Journal of Phase Equilibria 23 (2002).

[84] A Togo and I Tanaka, "First principles phonon calculations in materials science," Scr. Mater. 108, 1-5 (2015).

[85] A. Heiming, W. Petry, J. Trampenau, M. Alba, C. Herzig, and G. Vogl, "Phonons and martensitic phase transitions in pure bcc Ti and bcc Zr," Physical Review B 40, 11425-11428 (1989).

[86] Mario Burbano, Dany Carlier, Florent Boucher, Benjamin J. Morgan, and Mathieu Salanne, "Sparse cyclic excitations explain the low ionic conductivity of stoichiometric li $\mathrm{l}_{7} \mathrm{la}_{3} \mathrm{Zr}_{2} \mathrm{O}_{12}$," Phys. Rev. Lett. 116, 135901 (2016).

[87] Won-Seok Ko, Blazej Grabowski, and Jörg Neugebauer, "Development and application of a Ni-Ti interatomic potential with high predictive accuracy of the martensitic phase transition," Phys. Rev. B 92, 134107 (2015).

[88] Ambroise van Roekeghem, Jesús Carrete, and Natalio Mingo, "Anomalous thermal conductivity and suppression of negative thermal expansion in $\mathrm{ScF}_{3}$," Phys. Rev. B 94, 020303(R) (2016).

[89] Ion Errea, Matteo Calandra, and Francesco Mauri, "Anharmonic free energies and phonon dispersions from the stochastic self-consistent harmonic approximation: Application to platinum and palladium hydrides," Phys. Rev. B 89, 064302 (2014).

[90] Raffaello Bianco, Ion Errea, Lorenzo Paulatto, Matteo Calandra, and Francesco Mauri, "Second-order structural phase transitions, free energy curvature, and temperature-dependent anharmonic phonons in the selfconsistent harmonic approximation: Theory and stochastic implementation," Phys. Rev. B 96, 014111 (2017).

[91] Pavel Korotaev, Maxim Belov, and Aleksey Yanilkin, "Reproducibility of vibrational free energy by different methods," Computational Materials Science 150, 47 53 (2018).

[92] J. Towns, T. Cockerill, M. Dahan, I. Foster, K. Gaither, A. Grimshaw, V. Hazlewood, S. Lathrop, D. Lifka, G. D. Peterson, R. Roskies, J. R. Scott, and N. Wilkins-Diehr, "Xsede: Accelerating scientific discovery," Computing in Science \& Engineering 16, 62-74 (2014).

[93] "Appendix d - statistical errors," in Understanding Molecular Simulation (Second Edition), edited by Daan Frenkel and Berend Smit (Academic Press, San Diego, 2002) second edition ed., pp. 525 - 532.

[94] Christoph Freysoldt, Blazej Grabowski, Tilmann Hickel, Jörg Neugebauer, Georg Kresse, Anderson Janotti, and Chris G. Van de Walle, "First-principles calculations for point defects in solids," Rev. Mod. Phys. 86, 253-305 (2014).

[95] R. Nazarov, T. Hickel, and J. Neugebauer, "Vacancy formation energies in fcc metals: Influence of exchangecorrelation functionals and correction schemes," Phys. Rev. B 85, 144118 (2012).

[96] Ann E. Mattsson, Ryan R. Wixom, and Rickard Armiento, "Electronic surface error in the Si interstitial formation energy," Phys. Rev. B 77, 155211 (2008).

[97] Ann E. Mattsson, Rickard Armiento, Peter A. Schultz, and Thomas R. Mattsson, "Nonequivalence of the generalized gradient approximations PBE and PW91," Phys. 
Rev. B 73, 195123 (2006).

\title{
Supplemental Materials:
}

\section{Phonon-assisted diffusion in bcc phase of titanium and zirconium from first-principles}

\author{
Sara Kadkhodaei* and Ali Davariashtiyani \\ University of Illinois at Chicago, Chicago, Illinois, USA
}

(Dated: April 24, 2020)

\footnotetext{
* To whom correspondence should be addressed; Email: sarakad@uic.edu
} 


\section{SI. CALCULATION OF TEMPERATURE-DEPENDENT PHONON DISPER- SION AND DENSITY OF STATES}

Within the harmonic approximation, the Hamiltonian of the system is described as the following, for a system of $N$ particles

$$
\mathcal{H}=\frac{1}{2} \sum_{i=1}^{N} \sum_{\alpha=1}^{3}\left[\sqrt{M_{i}} \dot{u}_{i \alpha}\right]^{2}+\frac{1}{2} \sum_{i, j}^{N} \sum_{\alpha, \beta}^{3} \sqrt{M_{i}} u_{i \alpha} \frac{\Phi_{i, j}^{\alpha, \beta}}{\sqrt{M_{i} M_{j}}} \sqrt{M_{j}} u_{j \beta}
$$

where $u_{i \alpha}$ is the displacement of atom $i$ in the $\alpha$ direction, $M_{i}$ is the mass of atom $i$ and $\Phi$ is the $3 N \times 3 N$ force-constant matrix. Diagonalizing the dynamical matrix $\frac{\Phi_{i, j}^{\alpha, \beta}}{\sqrt{M_{i} M_{j}}}$ as

$$
\sum_{j}^{N} \sum_{\beta}^{3} \frac{\Phi_{i, j}^{\alpha, \beta}}{\sqrt{M_{i} M_{j}}} \epsilon_{\mu}^{j \beta}=\omega_{\mu}^{2} \epsilon_{\mu}^{i \alpha}
$$

results in obtaining the phonon frequencies, $\omega_{\mu}$ and eigenvectors, $\epsilon_{\mu}^{i \alpha}$. If the force-constant matrix is not positive-definite, some of the frequencies have imaginary values. We utilize two variations of the self-consistent harmonic approximation (SCHA) [1-5], which describes a harmonic model that minimizes the free energy rather than potential energy, which are significantly different in cases where harmonic phonon instabilities exist.

The self-consistent ab initio lattice dynamics (SCAILD) method [1-3] calculates atomic displacements based on an initial guess for phonon frequencies and accordingly updates the forces from first-principles calculation. A new set of phonon frequencies are obtained from the Fourier transformation of the updated atomic forces. This self-consistent process is performed until the difference in the vibrational free energy is converged to $10^{-5} \mathrm{eV}$, as shown in Fig. S1(b). Fig. S1(a) illustrates this iterative process performed on a $6 \times 6 \times 6$ supercell of bcc Ti including 216 atoms. The darkest dispersion curve in Fig. S1(a) corresponds to the harmonic phonon dispersion or zero-temperature phonon dispersion. The evolution of the phonon dispersion that includes the temperature-induced anharmonic effects is presented by smoothly varying the color of the curve from green to yellow (i.e., darkest to lightest). The converged phonon dispersion indicates no negative (imaginary) phonon frequencies as expected due to the dynamical stabilization of the phase at high enough temperature.

In the temperature-dependent effective potential (TDEP) method $[4,5]$, the temperaturedependent force-constant, $\tilde{\Phi}(T)$, is obtained by minimizing the difference between ab initio molecular dynamics (AIMD) forces and forces described in the harmonic approximation 


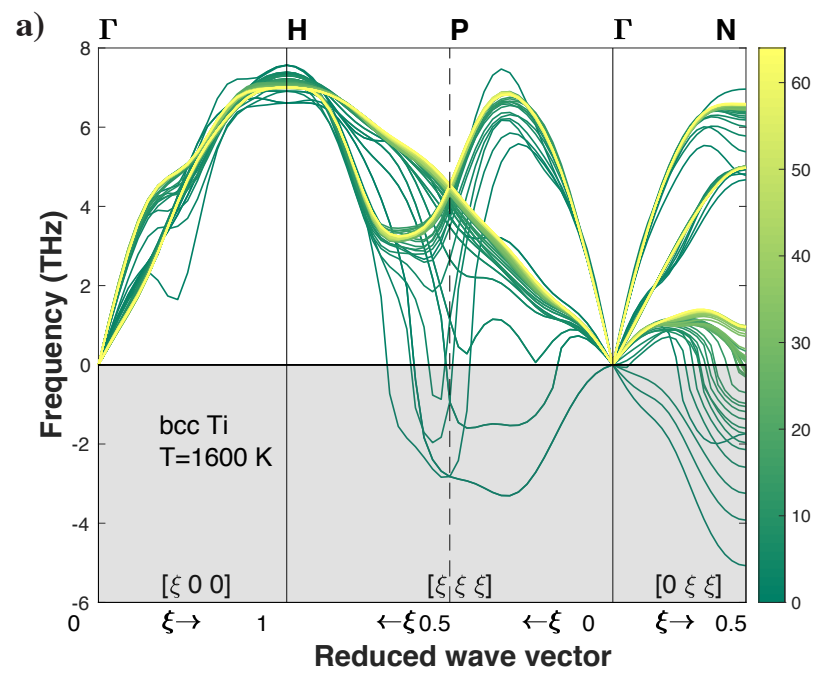

b)

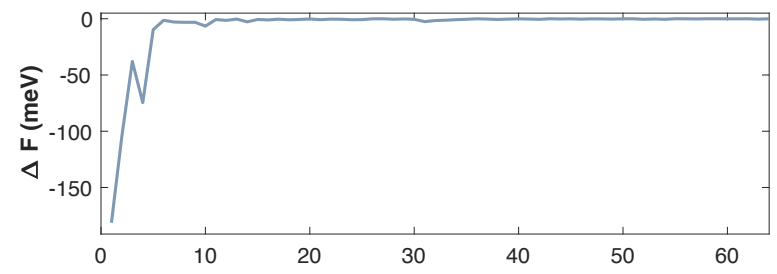

c)

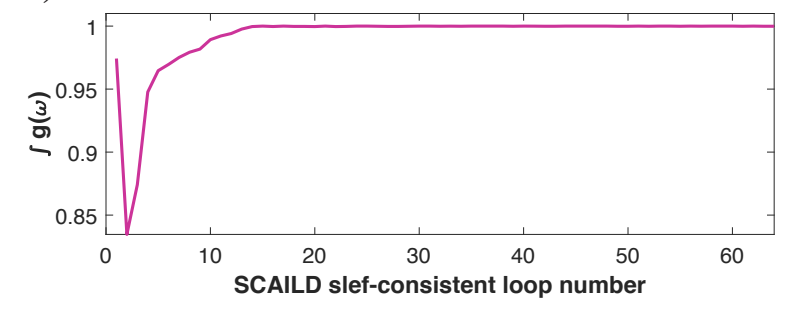

FIG. S1. a) Iterative evolution of anharmonic phonon dispersion for bcc Ti at $1600 \mathrm{~K}$, obtained from the SCAILD method. The color bar indicates the iterative steps. The darkest color indicates the initial step, where the phonon dispersion is calculated using the harmonic approximation, as opposed to the lightest color which indicates the final step. b) The vibrational free energy difference, $\Delta F$, between consecutive SCAILD steps. The iterative steps are performed until $\Delta F$ is smaller than $10^{-5} \mathrm{eV}$. c) The evolution of the integral of phonon density of states along the SCAILD iterative steps.

according to the following equation,

$$
\min \frac{1}{N_{t}} \sum_{t=1}^{N_{t}}\left\|f_{t}^{A I M D}-f_{t}^{H}\right\|=\min _{\tilde{\Phi}(T)} \frac{1}{N_{t}} \sum_{t=1}^{N_{t}}\left\|f_{t}^{A I M D}-\tilde{\Phi}(T) u_{t}^{A I M D}\right\|
$$

where $\left\{f_{t}^{A I M D}, u_{t}^{A I M D}\right\}$ are the set of AIMD forces and displacements obtained from the AIMD trajectory of length $N_{t}$. We performed AIMD simulations for each temperature using an NVT ensemble under the Nose-Hoover thermostat formalism [6-10]. The temperaturedependent force constant tensor is obtained by least square fitting of AIMD forces and displacements to a harmonic model according to Eq. 3 using our in-house code. The AIMD forces and displacement are captured every 0.01 ps in a trajectory of 35 ps, after thermalization of the system for 1 ps (for details on the burning and decay time see the next section). Fig. S2 illustrates the anharmonic phonon dispersion and density of states for bcc Ti in the range of 1300 to $1600 \mathrm{~K}$, obtained from temperature-dependent force constant tensors using the Phonopy code [11]. There is a slight difference between the dispersion curves obtained 
from SCAILD and TDEP, which stems from the different approaches taken in these methods to optimize the effective harmonic model to the Hamiltonian in Eq. 1. In SCAILD, the iterative steps are taken in the frequency-polarization space (the Fourier transformed of time-space pair) by assuming that the polarization vectors are constant and only phonon frequencies are optimized (see Eq. 2). However, in TDEP the polarization vectors are optimized according to temperature-induced effects when the force-constant tensor in Eq. 1 is fitted to AIMD forces and displacements.

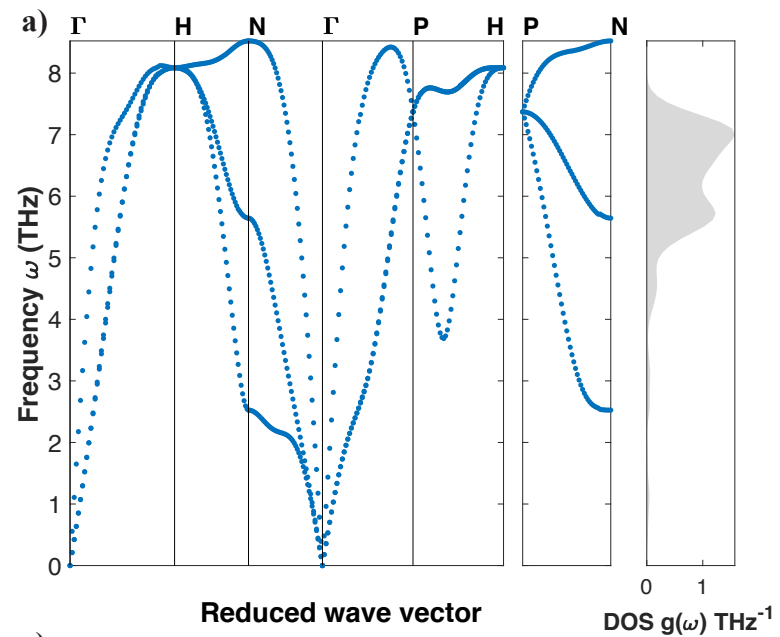

b)
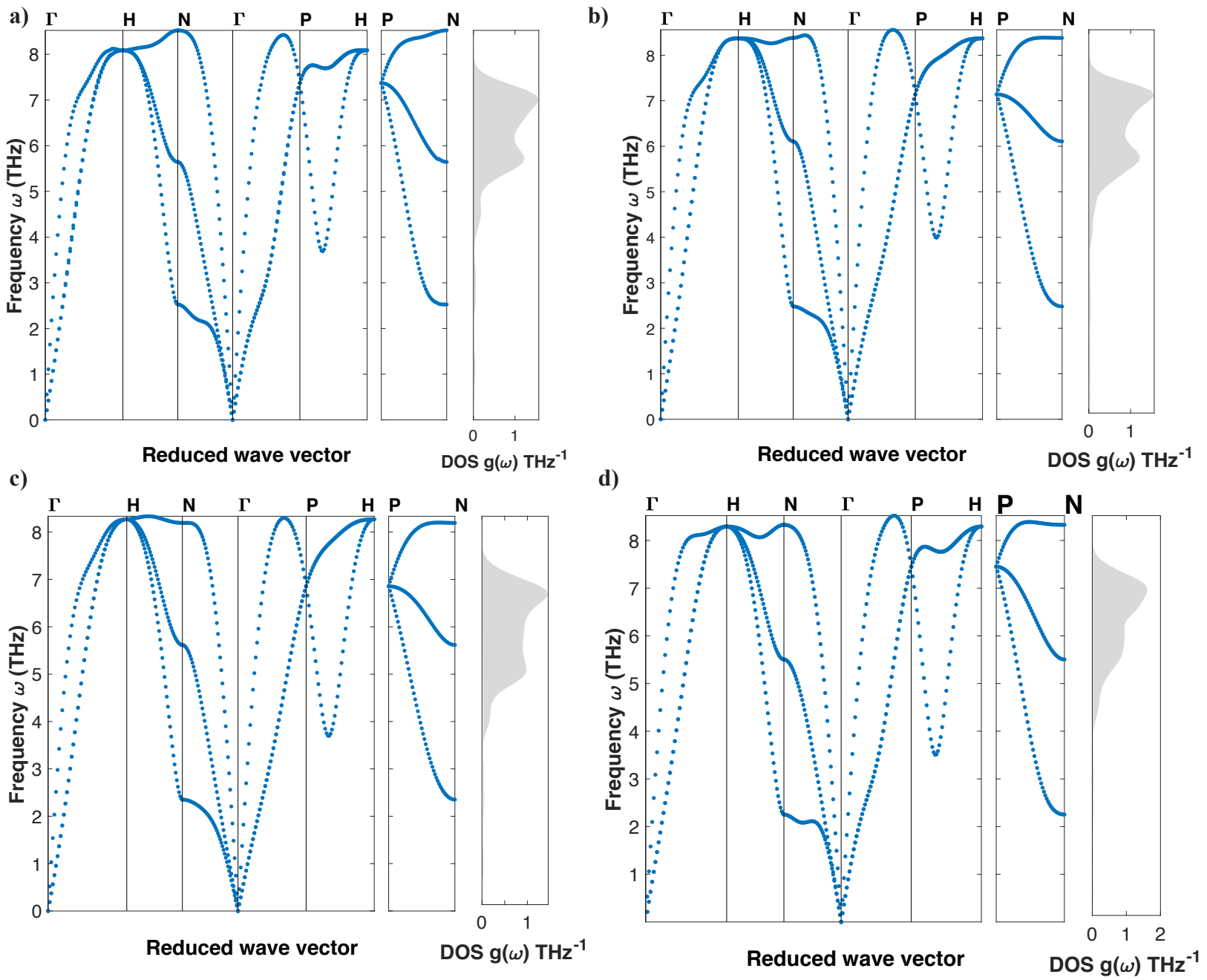

FIG. S2. a) The anharmonic phonon dispersion and density of states for the bulk bec Ti at a) $1300 \mathrm{~K}, \mathrm{~b}) 1400 \mathrm{~K}, \mathrm{c}) 1500 \mathrm{~K}$ and d) $1600 \mathrm{~K}$, obtained from the TDEP method. 


\section{SII. CALCULATION OF VACANCY FORMATION ENTHALPY}

Since the bcc phase of IIIB and IVB metals is a high-temperature phase, the total DFT energy at zero temperature is not a good approximation for the enthalpy of the system, while it is usually used for mechanically stable phases. We obtain the enthalpy of the bulk or defected bcc phase as the following

$$
H=\langle U\rangle+\langle p\rangle V,
$$

where $\langle U\rangle$ is the NVT ensemble average of the internal energy, consisting of the potential and kinetic energy at each temperature, $\langle p\rangle$ is the average pressure, and $\mathrm{V}$ is the total volume of the system. The canonical ensemble averages are obtained by block averaging of AIMD internal energy and pressure according to the scheme described in appendix D.3 of Ref. [12]. To ensure that the energy and pressure values are obtained from uncorrelated MD snapshots, the characteristic decay time of the MD trajectory is obtained from the limit of the following product function, $P\left(t_{B}\right)$, when $t_{B} \rightarrow \infty[12]$.

$$
P\left(t_{B}\right)=t_{B} \times \frac{\sigma^{2}\left(\overline{A_{B}}\right)}{\left\langle A^{2}\right\rangle-\langle A\rangle^{2}}
$$

Here, $\overline{A_{B}}$ is the block average of any observable A (i.e., the time average over a finite time $\left.t_{B}\right), \sigma^{2}$ is the variance of the block average, and $\langle A\rangle$ is the ensemble average of $\mathrm{A}$. In the limit of block time being much larger than the characteristic decay time of the observable time correlation function, $\mathrm{P}$ will approach the characteristic decay time (refer to Ref. [12] for more details). Fig. S3(b) shows the $P\left(t_{B}\right)$ function as a function of block time, obtained by time averaging the internal energy $U$ from the AIMD trajectory. For a block time larger than $0.2 \mathrm{ps}$, the $\mathrm{P}$ value does not change dramatically and merely oscillates around 0.015 ps, which is the characteristic decay time of the AIMD time correlation function. As shown in Fig. S3(a), the time required for the Nose-Hoover thermostat to equilibrate is determined to be $1 \mathrm{ps}$ according to the temperature-time curve. The average internal energy and pressure for each system at each temperature are obtained by averaging the AIMD snapshots internal energy and pressure captured every 0.1 ps (larger than the decay time) after the burning equilibration time, as shown in Fig. S3(c-d). The vacancy formation enthalpy is then calculated as

$$
\Delta H_{v}=H^{t o t}(N-1)-\frac{N-1}{N} H^{t o t}(N),
$$



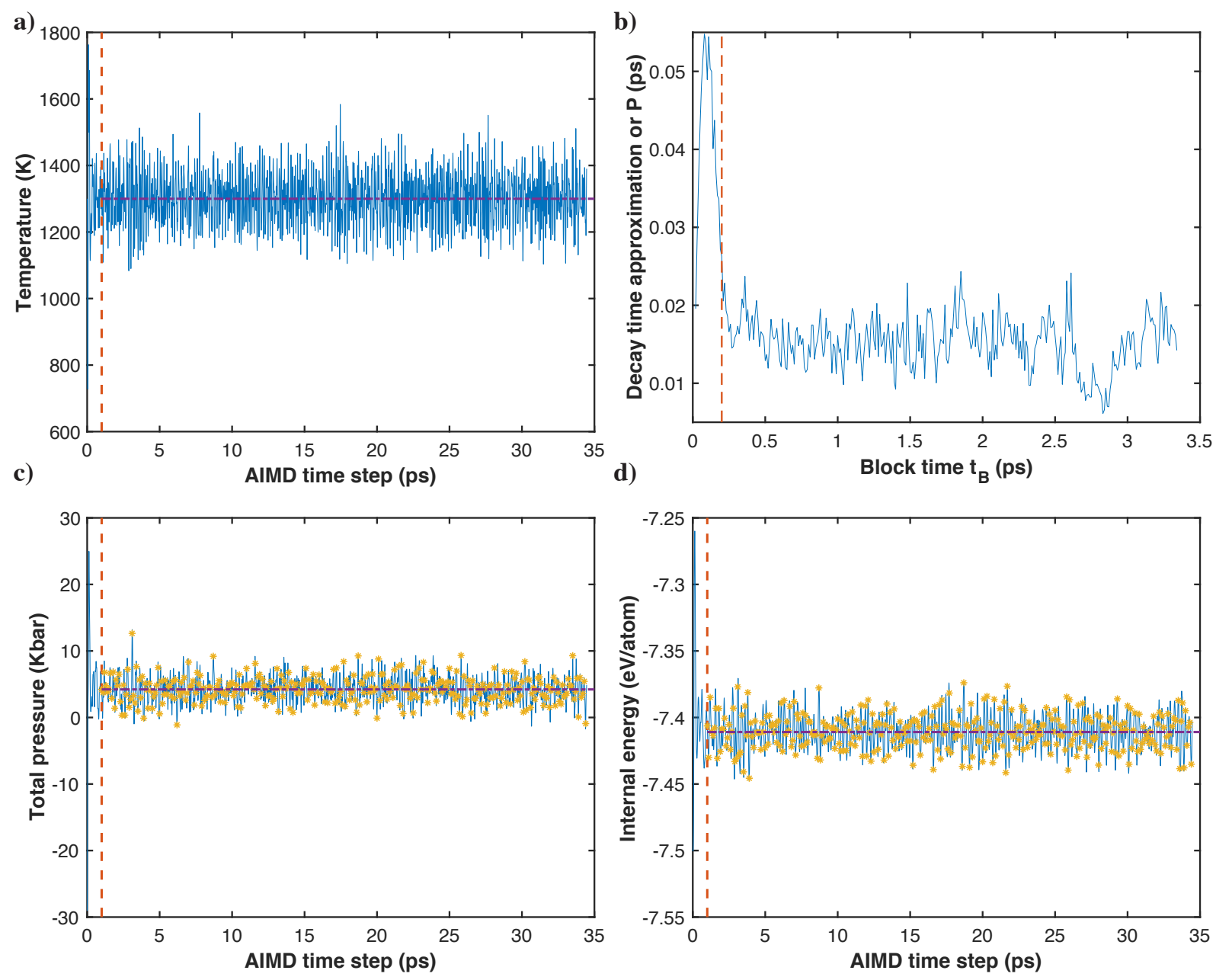

FIG. S3. a) Temperature vs. time for the canonical molecular dynamics simulation of bulk bcc $\mathrm{Ti}$ at $1300 \mathrm{~K}$ using the Nose-Hoover thermostat. The thermalization time (a.k.a. the burning time) is illustrated by the dashed line. b) The $\mathrm{P}$ value, $t_{B} \times \frac{\sigma^{2}\left(\bar{U}_{B}\right)}{\left\langle U^{2}\right\rangle-\langle U\rangle^{2}}$, for the MD trajectory as a function of the block time, $t_{B}$. For a block time larger than $0.2 \mathrm{ps}$ (dashed line), the $\mathrm{P}$ value does not change and merely oscillates around $0.015 \mathrm{ps}$, which is the characteristic decay time. c-d) The AIMD pressure and internal energy vs. time. The star marks indicate the values captured for statistical averaging and the dotted lines are the mean values.

where $H^{\text {tot }}(N-1)$ and $H^{\text {tot }}(N)$ are the enthalpy for the bulk and defected systems, respectively. A correction term is added to the vacancy formation enthalpy as described in the next section. 

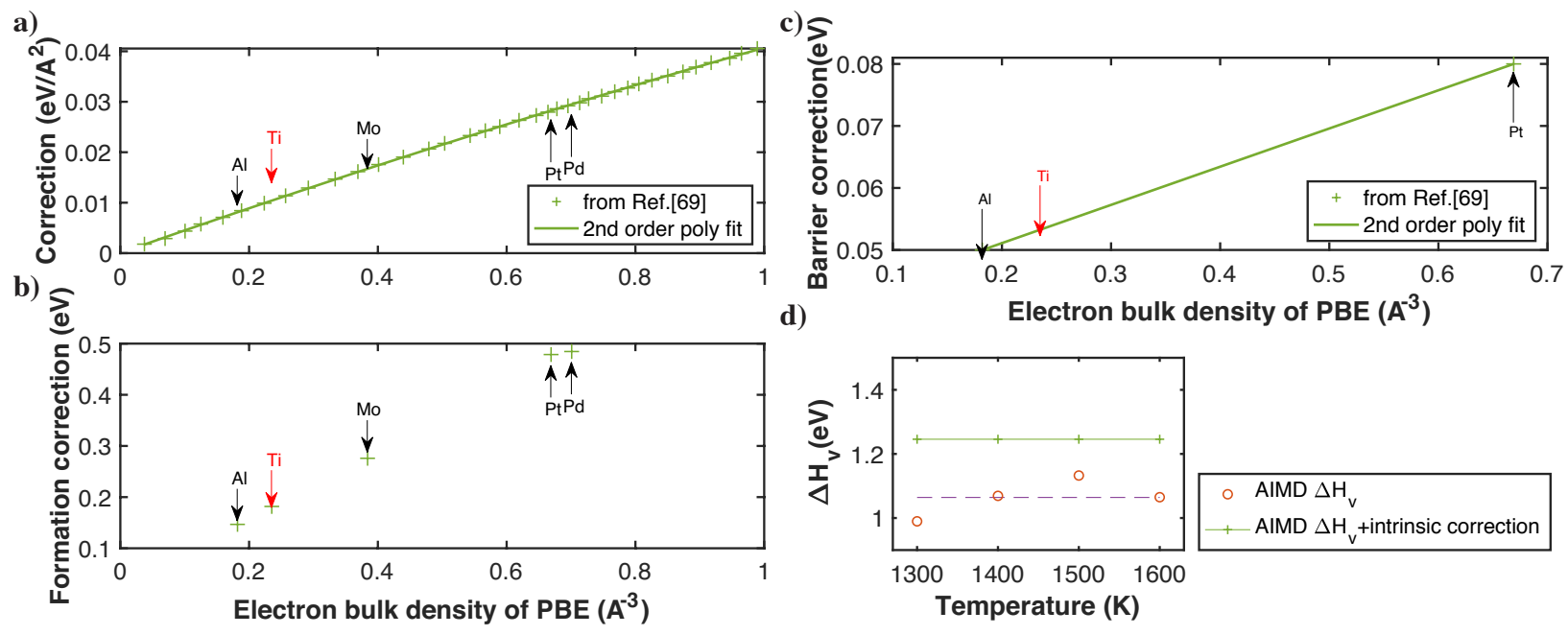

FIG. S4. a) The intrinsic surface correction term per area as a function of bulk valence electron density of the PBE exchange-correlation functional. Cross values are extracted from Fig. 5 of Ref. [13]. b) The total correction term for vacancy formation energy for $\mathrm{Ti}, \mathrm{Al}, \mathrm{Mo}, \mathrm{Pt}$, and Pd. c) The total correction term for vacancy migration energy for $\mathrm{Ti}, \mathrm{Al}$ and $\mathrm{Pt}$. d) The vacancy formation enthalpy in bcc $\mathrm{Ti}$ as a function of temperature. The circles are the statical average formation enthalpy obtained from AIMD simulation using the PBE exchange-correlation functional. The crosses are the formation enthalpy values including the explicit intrinsic surface correction term.

\section{SIII. CALCULATION OF INTRINSIC SURFACE CORRECTION TERM FOR THE VACANCY FORMATION AND MIGRATION ENTHALPIES}

It has been shown in the literature that density functional theory underestimates the vacancy formation and migration enthalpies [13-18]. This underestimation has been associated to electron correlation effects near electronic edges of the intrinsic surface which is formed due to vacancy formation [14], and is predominantly a function of bulk electron density for both the LDA and GGA exchange-correlation functional formalism [13]. Fig. S4(a) illustrates the explicit correction term per area as a function of the bulk electron density for the PBE exchange-correlation functional [19] extracted from Fig.5 of Ref. [13]. We used the PBE exchange-correlation functional as implemented in PAW-PBE pseudopotential of VASP [20]. The number of valence electrons for each conventional bcc unit cell is $2 N_{\text {elect }}=2 \times 4$ and the bulk electron density is $\rho=\frac{2 N_{\text {elect }}}{a_{0}^{3}}=0.235 \AA^{-3}$, where $a_{0}=3.24$. We calculated the exposed surface area of vacancy in bcc Ti to be $17.46 \AA^{2}$, based on the vacancy radius in 
fcc $\mathrm{Al}$ and the scaling scheme described in table III of Ref. [13]. Fig. S4(b) illustrate the vacancy formation correction term for bcc Ti alongside the correction values for $\mathrm{Al}$, Mo, $\mathrm{Pt}$, and Pd [13]. We calculate the final correction term by multiplying the correction term per area in Fig. S4(a) by the exposed vacancy surface in each element, which is in good agreement with values presented in table III of Ref. [13]. The migration barrier energy is calculated more accurately than the formation energy in DFT, simply because two more similar energies are compared (i.e., propagation of surface formation error along the transition path) than when calculating the formation energy [13]. As illustrated in Fig. S4(c), we

calculated the explicit correction term for migration energy by interpolating the results for fcc Al and fcc Pt extracted from Ref, [14] and Ref. [13], respectively. Fig. S4(d) indicates the AIMD formation enthalpy and the final formation enthalpy which includes the intrinsic surface correction term.

\section{SIV. CALCULATION OF VACANCY FORMATION ENTROPY AND EQUILIB- RIUM MONOVACANCY CONCENTRATION}

The vacancy formation entropy is calculated at each temperature according to the following equation:

$$
\Delta S_{v}=S^{t o t}(N-1)-\frac{N-1}{N} S^{t o t}(N),
$$

where $N$ is the number of atoms in the supercell and $S^{\text {tot }}(N-1)$ and $S^{\text {tot }}(N)$ are the vibrational entropy for bcc with a monovacancy and bulk bcc, respectively. The vibrational entropy is calculated given the anharmonic and temperature-dependent phonon frequencies and density of states according to the following equation:

$$
S_{v i b}=-k_{B} \int_{0}^{\infty} \ln \left[2 \sinh \left(\frac{\hbar \omega(T)}{2 k_{B} T}\right)\right] g(\omega(T)) d \omega,
$$

where $\omega(T)$ and $g(\omega(T))$ are temperature-dependent phonon frequencies and density of states, respectively. The vacancy formation entropy for bcc Ti at each temperature is shown in Fig.4(b) in the main text and for bcc Zr is shown in Fig. S11. The equilibrium monovacancy concentration, $C_{v}$, is calculated according to

$$
C_{v}=\exp \left(-\frac{\Delta H_{v}}{k_{B} T}\right) \exp \left(\frac{\Delta S_{v}}{k_{B}}\right),
$$

where $\Delta H_{v}$ and $\Delta S_{v}$ are enthalpy and entropy of monovacancy formation, respectively. The monovacancy concentration for bcc Ti obtained according to the anharmonic phonon 
analysis is presented in Fig. 4 in the main text (similar plot for bcc $\mathrm{Zr}$ is presented in Fig. S11). We also calculate the vacancy formation entropy and concentration for the case where the harmonic phonon analysis is used (and existing imaginary phonon frequencies are ignored). As illustrated in Fig. 4 of the main text and Fig. S11, in the harmonic approximation the formation entropy is independent of temperature, since it is obtained based on temperature-independent phonon frequencies and DOS at $0 \mathrm{~K}$.

\section{SV. CALCULATION OF DIFFUSION COEFFICIENT FOR BCC ZIRCONIUM}

To confirm the validity of the proposed approach, we calculate the diffusion coefficient for bcc $\mathrm{Zr}$ in addition to bcc Ti. In the following, we elaborate on diffusivity calculation for bcc Zr, which is conducted in the same way as bcc Ti explained in the main letter. Fig S5 illustrates the zero-temperature phonon dispersion, density of states, and total energy for a $6 \times 6 \times 6$ supercell of bcc $\mathrm{Zr}$ with a monovacancy (the lattice parameter is $a_{0}=3.59 \AA$ ). The dispersion and DOS are obtained from DFT forces and displacements according to the finite displacement approach implemented in the Vienna Ab-initio Simulation Package (VASP) [20-23]. The projector augmented wave (PAW) method [24, 25] with energy cutoff of $229.9 \mathrm{eV}$ and the generalized gradient approximation (GGA) [20, 23, 26] with the PBE [19] exchange-correlation are used. The pseudo-potential includes 12 electrons in the valance shell (the $\mathrm{Zr}$ ssv as implemented in VASP). As shown in Fig. S6, the converged temperaturedependent phonon dispersion for bcc $\mathrm{Zr}$ is obtained at the range of $1300 \mathrm{~K}$ to $1700 \mathrm{~K}$ according to the SCAILD method explained in section SI. The effective vibration frequency along the vacancy diffusive path, $\nu^{*}$, is assumed to be the frequency of the $2 / 3 L\langle 111\rangle$ phonon mode, as shown by the star mark in Fig. S6(d). Fig. S7 indicates the T-dependent phonon dispersions for both the bulk bcc zirconium and the bcc Zr including a vacancy obtained by the TDEP method as explained in section SI. The AIMD forces and displacement are captured every $0.02 \mathrm{ps}$ in a trajectory of $35 \mathrm{ps}$, after thermalization of the system for $1 \mathrm{ps}$ (for details on the burning and decay time Fig. S8). The vacancy formation enthalpy is obtained according to Eq. 6, where the enthalpy for the bulk and defected systems are obtained form AIMD energy and pressure averages according to Eq. 4. For statistical averaging, we capture AIMD energy and pressure every 0.1 ps (above the 0.02 ps decay time shown in Fig S8(d)) and after the 1 ps thermalization time as shown in Fig S8(a). The vacancy formation 
enthalpy from average AIMD calculation is explicitly corrected for the "intrinsic surface" error of DFT, as explained in section SIII. Moreover, the migration enthalpy is approximated as the DFT energy difference of the initial and transition states, as illustrated in Fig S5(c-d). The migration energy is also corrected for the internal surface effect according to details of section SIII. Fig. S9 indicates the correction terms and corrected vacancy formation enthalpy for bcc Zr.

Fig. S10 indicates the T-dependent phonon density of states for the bulk and defected bcc Zr, obtained by the TDEP method. The vibrational entropy for each system is obtained according to Eq. 8 and the vacancy formation entropy is then obtained according to Eq. 7. Fig. S11 shows the formation entropy and equilibrium concentration for a monovacancy in bcc $\mathrm{Zr}$ obtained according to the anharmonic vibrational analysis. It is compared with the results obtained by a standard harmonic treatment of lattice vibration as explained in section SIV. 
a)

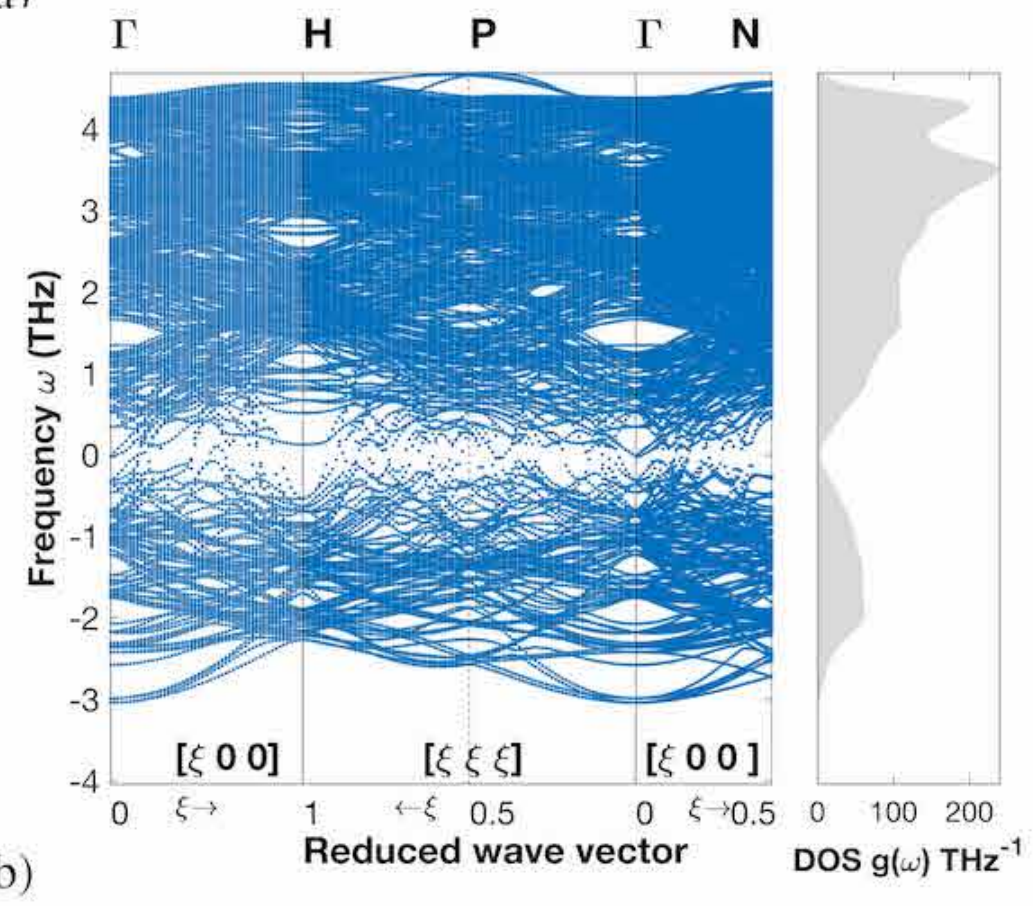

c)

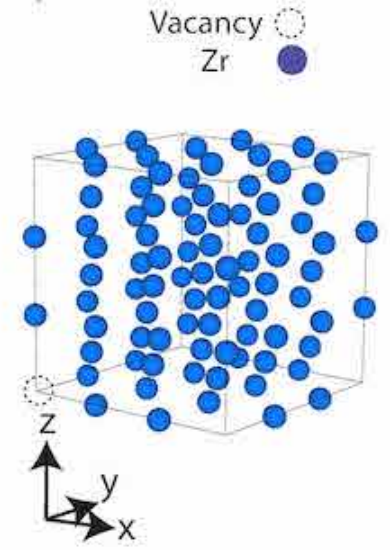

total energy $=-1805.93 \mathrm{eV}$

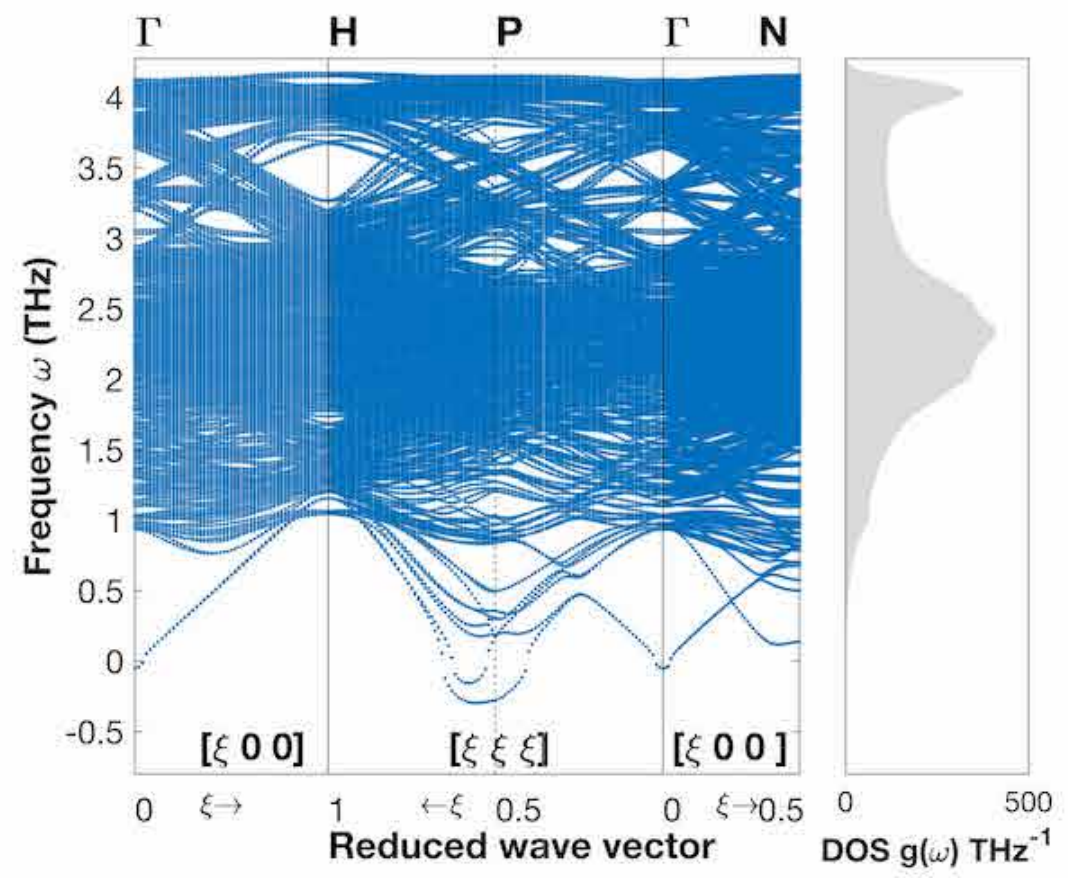

d)
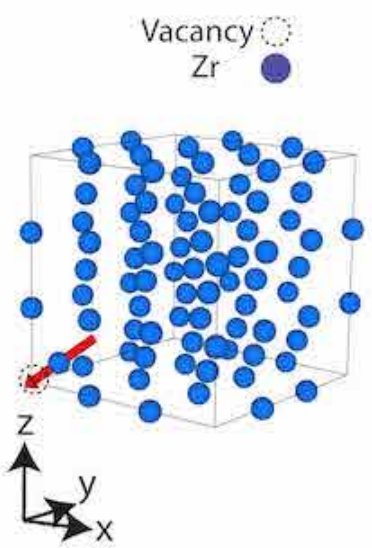

total energy $=-1805.53 \mathrm{eV}$

FIG. S5. DFT harmonic phonon dispersion and density of states (DOS) for a $6 \times 6 \times 6$ supercell of bcc zirconium including 215 atoms and a monovacancy a) at the bcc site, and b) migrated half way to the nearest neighbor bcc site along [111] direction. Phonon instabilities are depicted as negative frequencies in the dispersion curves. The atomic configuration for the initial and intermediate states of vacancy diffusion are illustrated in c) and d), respectively. A conventional supercell is presented for visualization simplicity. 
a)

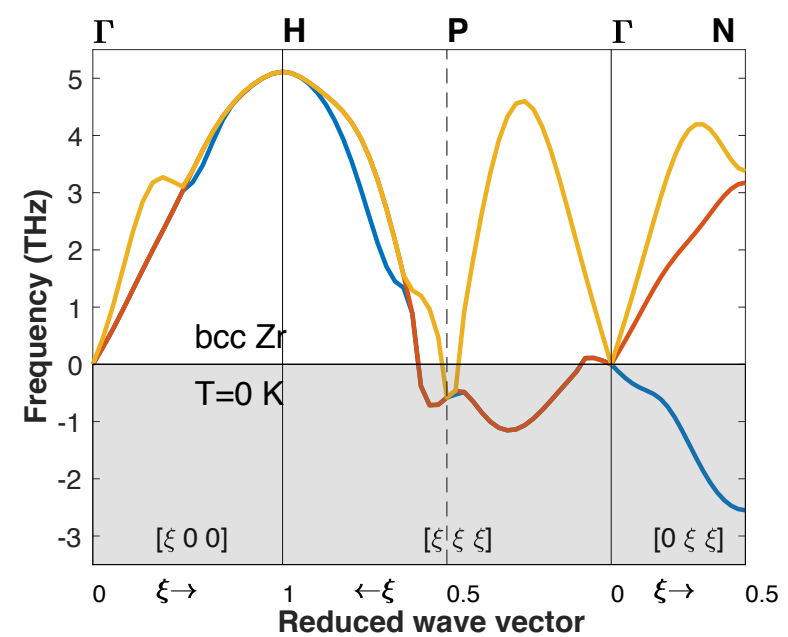

c)

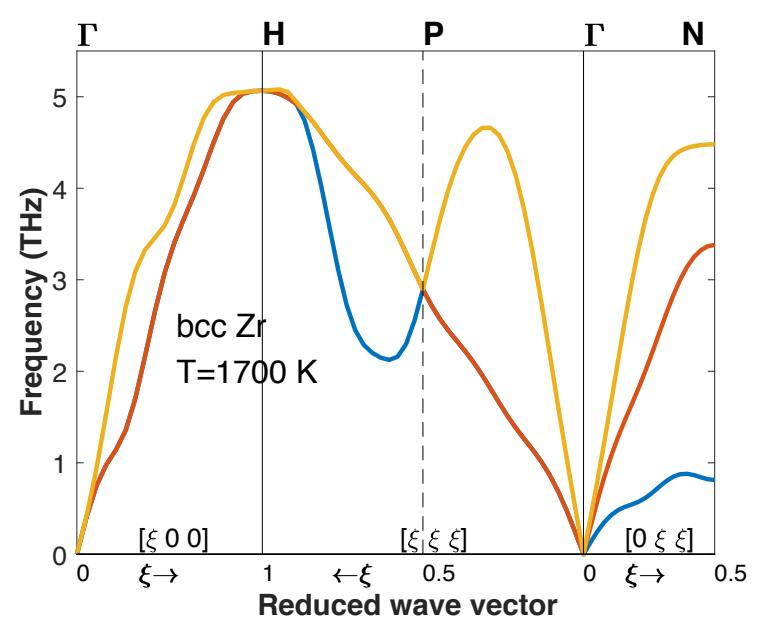

b)

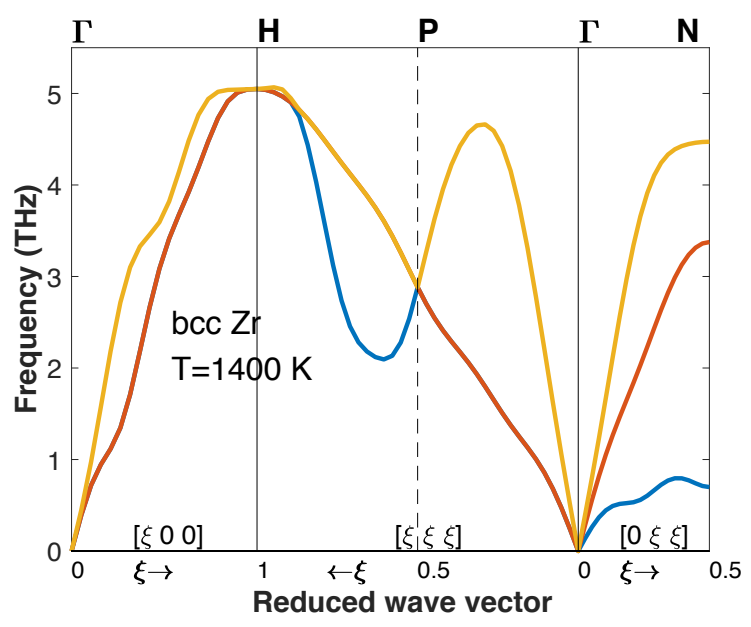

d)

e)

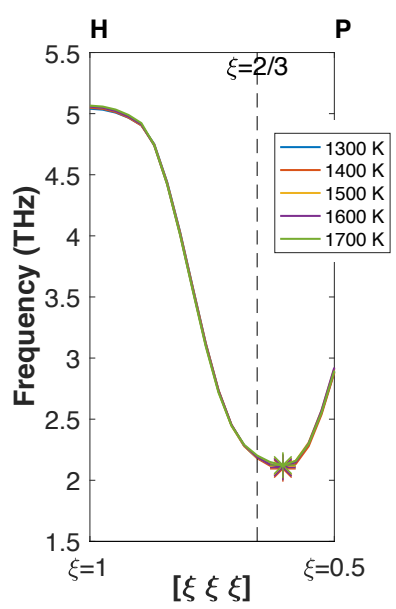

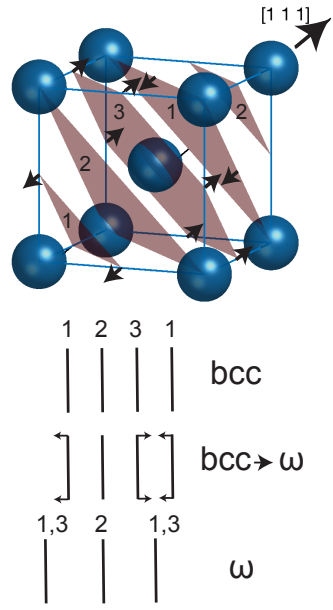

FIG. S6. Phonon dispersion for a bulk defect-free $6 \times 6 \times 6$ supercell of bcc zirconium including 216 atoms at a) $0 \mathrm{~K}$, b) $1400 \mathrm{~K}$, and c) $1700 \mathrm{~K}$. d) The longitudinal phonon branch along the $[\xi \xi \xi]$ reduced wave vector (between $\mathrm{H}$ and $\mathrm{P}$ high-symmetry points) from $1300 \mathrm{~K}$ to $1700 \mathrm{~K}$. The star marks in (d) represent the negative dip on the phonon branch. e) The trigonal distortion of (111) atomic planes along the [111] direction associated with the eigenvector of $\xi=\frac{2}{3}$ phonon mode along the $\Gamma-\mathrm{P}-\mathrm{H}$ branch. A complete collapse of atomic planes 1 and 3 results in the hexagonal $\omega$ phase. 
a) bulk bcc $\mathrm{Zr}(\mathrm{T}=1500 \mathrm{~K})$

b) bcc Zr with a monovacancy ( $T=1500 \mathrm{~K})$

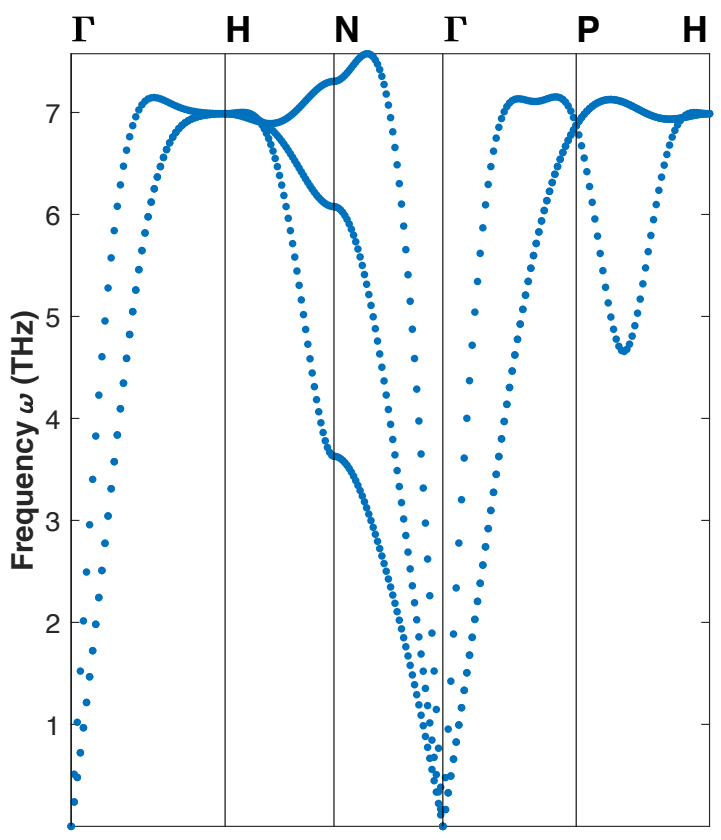

Reduced wave vector

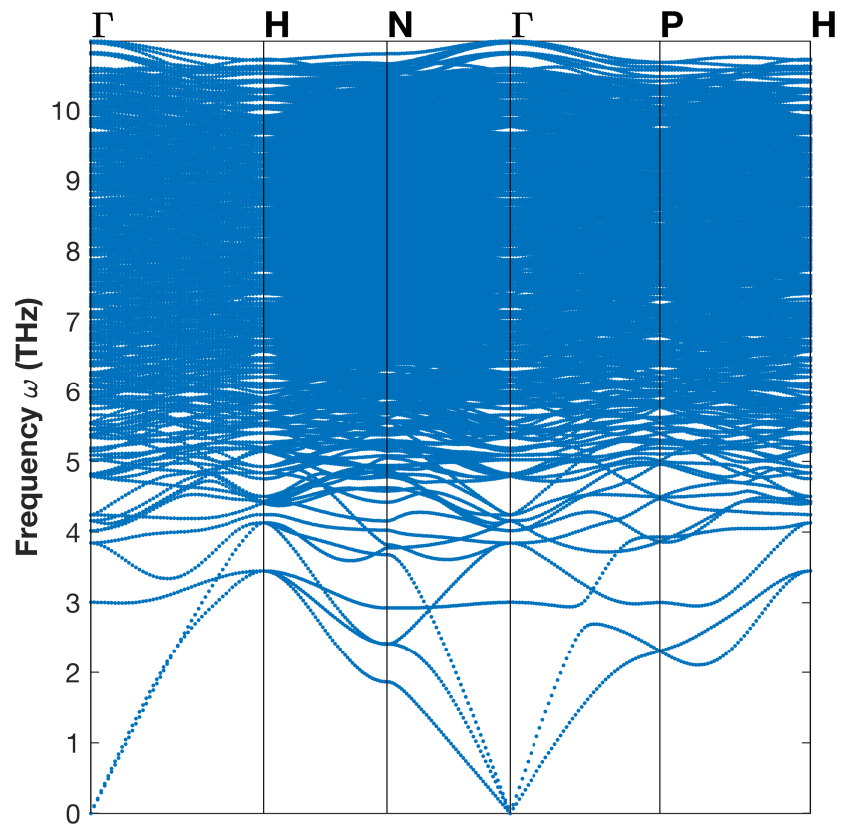

Reduced wave vector

FIG. S7. The anharmonic phonon dispersion for a) a $6 \times 6 \times 6$ supercell of bcc zirconium including 216 atoms and b) bcc Zr with a monovacancy including 215 atoms at $1500 \mathrm{~K}$. The phonon dispersion and DOS for the defected system are obtained by enforcing the bcc crystal symmetry. 
a)

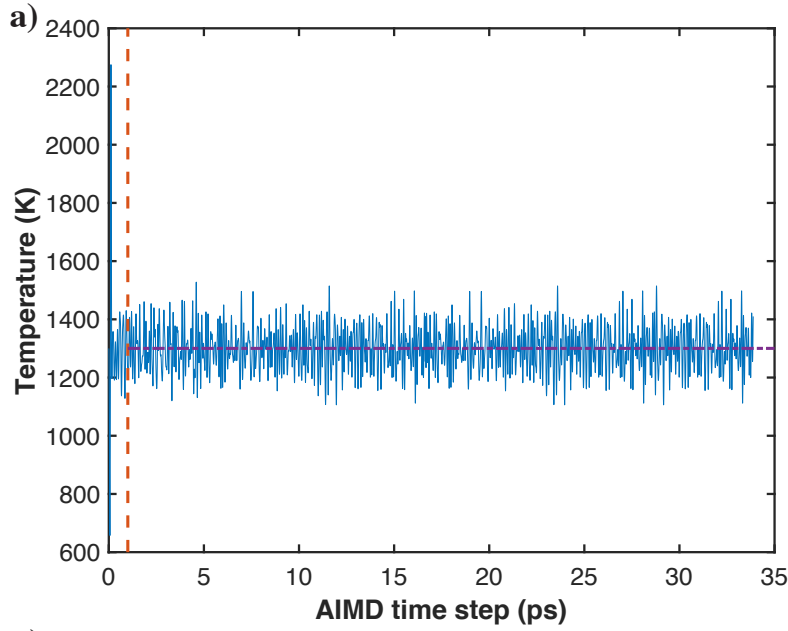

c)

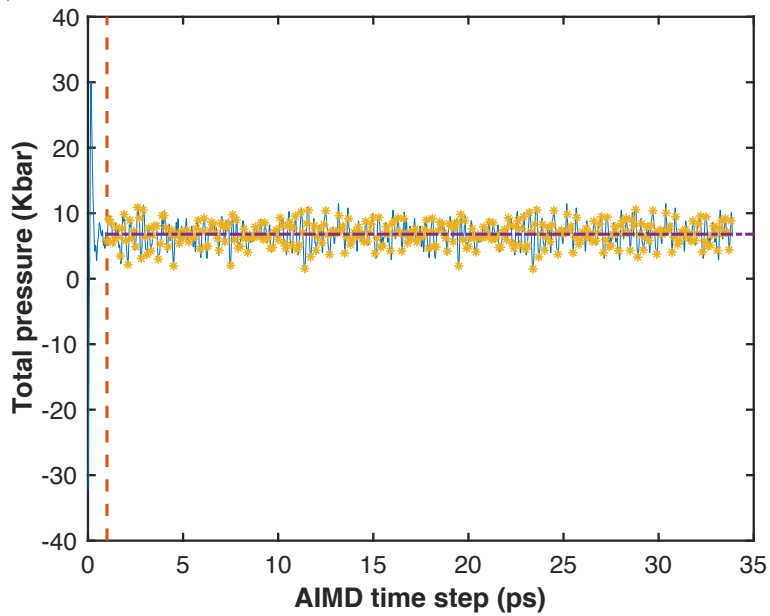

b)

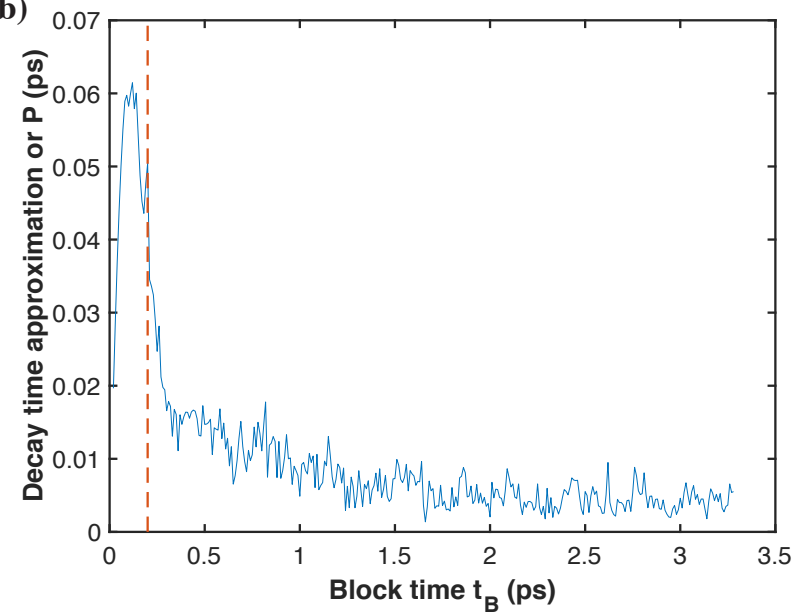

d)

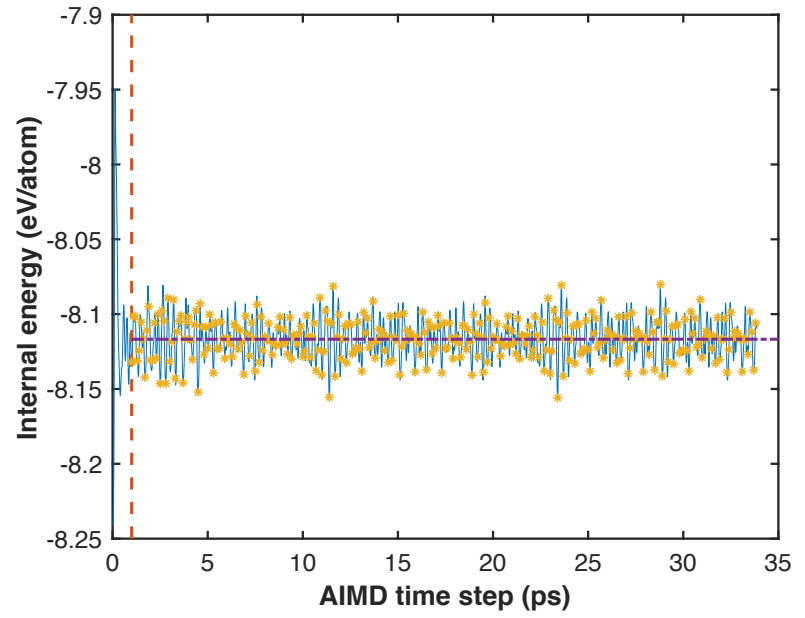

FIG. S8. a) Temperature vs. time for the canonical molecular dynamics simulation of bulk bcc Zr at $1300 \mathrm{~K}$ using the Nose-Hoover thermostat. The thermalization time (a.k.a. the burning time) is illustrated by the dashed line. b) The $\mathrm{P}$ value, $t_{B} \times \frac{\sigma^{2}\left(\bar{U}_{B}\right)}{\left\langle U^{2}\right\rangle-\langle U\rangle^{2}}$, for the MD trajectory as a function of the block time, $t_{B}$. For a block time larger than $0.2 \mathrm{ps}$ (dashed line), the $\mathrm{P}$ value does not change and merely oscillates around $0.005 \mathrm{ps}$, which is the characteristic decay time. c-d) The AIMD pressure and internal energy vs. time. The star marks indicate the values captured for statistical averaging. 


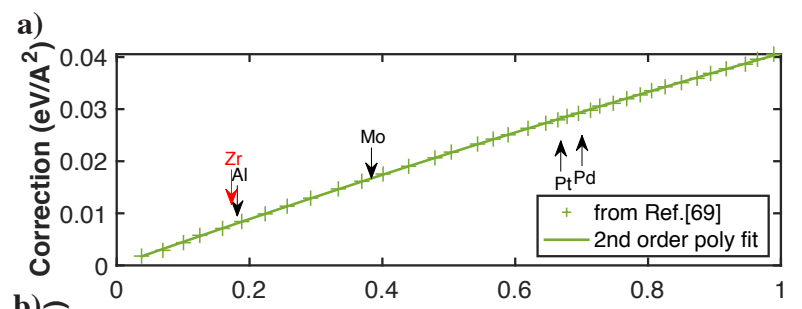

b) $>$

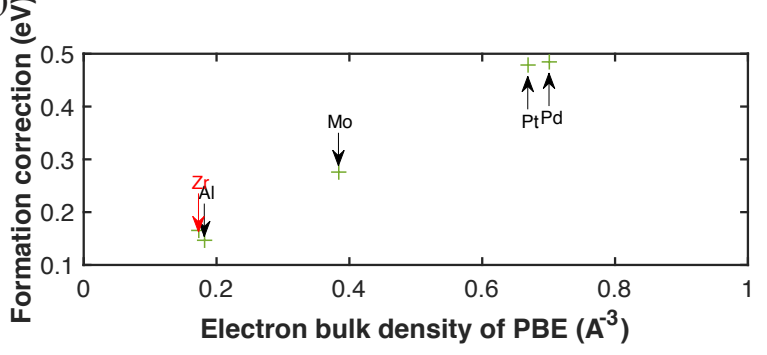

c)

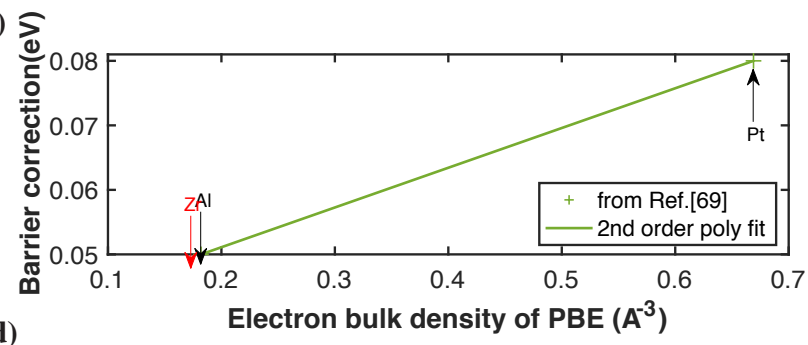

d)

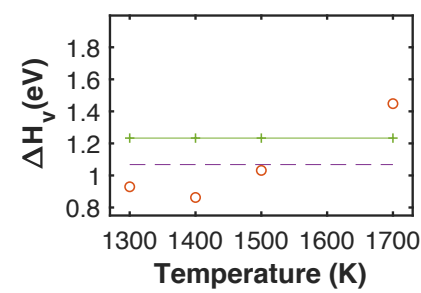

- AIMD $\Delta \mathrm{H}_{\mathrm{v}}$

AIMD $\Delta \mathrm{H}_{\mathrm{v}}+$ intrinsic correction

FIG. S9. a) The intrinsic surface correction term per area as a function of bulk valence electron density of the PBE exchange-correlation functional. Cross values are extracted from Fig. 5 of Ref. [13]. b) The total correction term for vacancy formation energy for Zr, Al, Mo, Pt, and Pd. c) The total correction term for vacancy migration energy for $\mathrm{Zr}, \mathrm{Al}$ and $\mathrm{Pt}$. d) The vacancy formation enthalpy in bcc Zr as a function of temperature. The circles are the statical average formation enthalpy obtained from AIMD simulation using the PBE exchange-correlation functional. The crosses are the formation enthalpy values including the explicit intrinsic surface correction term.

a)

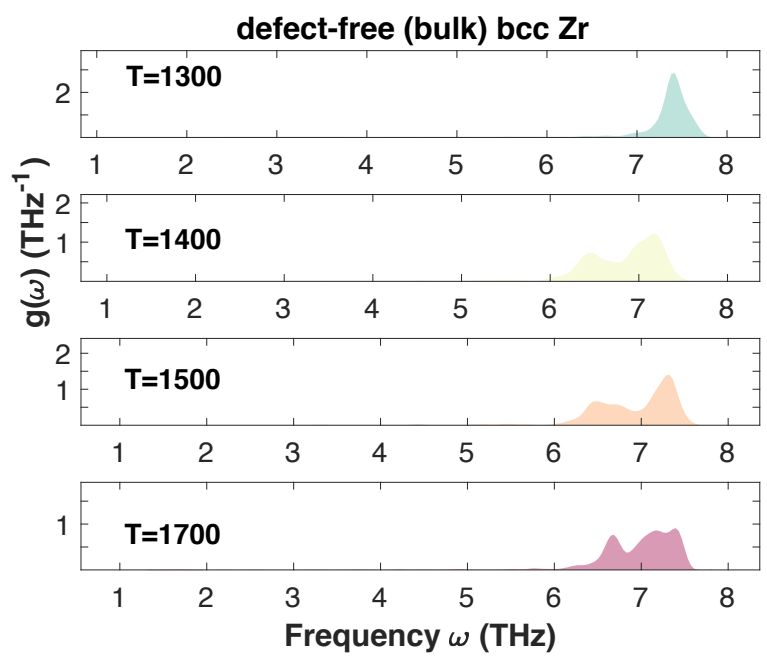

b)
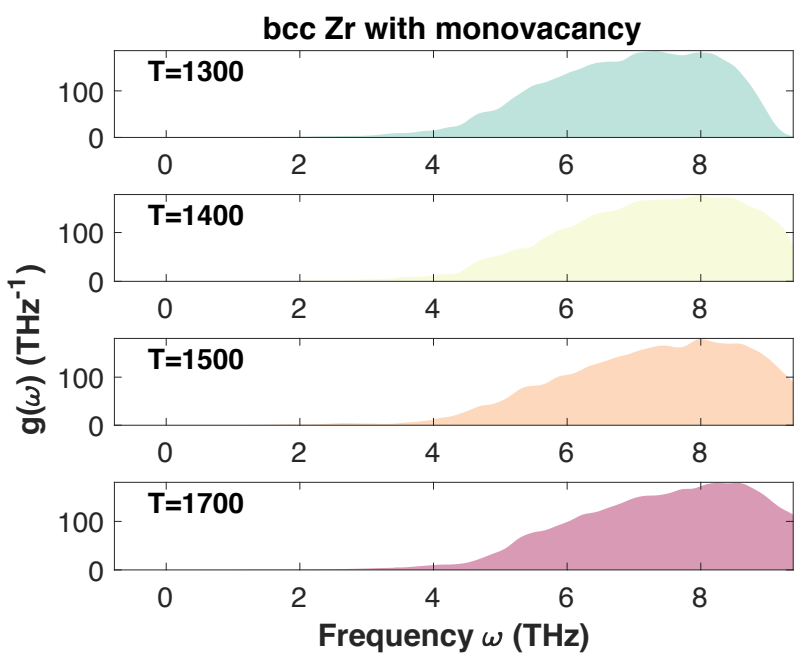

FIG. S10. The anharmonic phonon density of states for bulk bcc Zr (the left plots) and the bcc phase with a monovacany (the right plots) at different temperatures. 

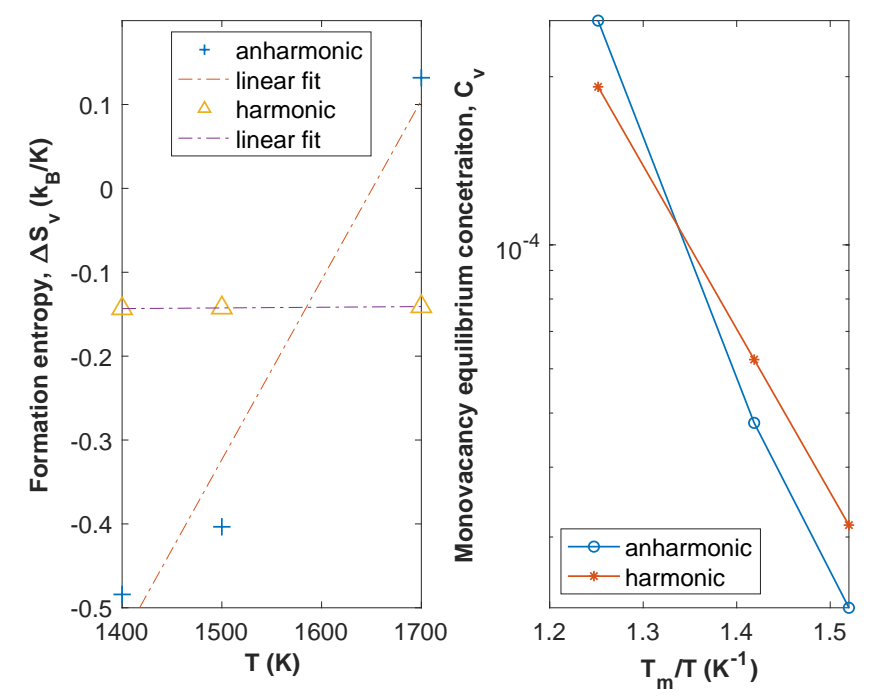

FIG. S11. The formation entropy and equilibrium concentration versus temperature for monovacany in bcc $\mathrm{Zr}$. $\mathrm{Tm}=2153 \mathrm{~K}$. 
[1] N. R. Werthamer, "Self-Consistent Phonon Formulation of Anharmonic Lattice Dynamics," Physical Review B 1, 572-581 (1970).

[2] P. Souvatzis, O. Eriksson, M. I. Katsnelson, and S. P. Rudin, "Entropy Driven Stabilization of Energetically Unstable Crystal Structures Explained from First Principles Theory," Physical Review Letters 100, 095901 (2008).

[3] P. Souvatzis, O. Eriksson, M.I. Katsnelson, and S.P. Rudin, "The self-consistent ab initio lattice dynamical method," Computational Materials Science 44, 888 - 894 (2009).

[4] O. Hellman, I. A. Abrikosov, and S. I. Simak, "Lattice dynamics of anharmonic solids from first principles," Physical Review B 84, 180301(R) (2011).

[5] Olle Hellman, Peter Steneteg, I. A. Abrikosov, and S. I. Simak, "Temperature dependent effective potential method for accurate free energy calculations of solids," Physical Review B 87, 104111 (2013).

[6] William G. Hoover and Brad Lee Holian, "Kinetic moments method for the canonical ensemble distribution," Physics Letters A 211, 253 - 257 (1996).

[7] S. Nosé, "A molecular dynamics method for simulations in the canonical ensemble," Molecular Physics 52, 255-268 (1984).

[8] Shuichi Nosé, "A unified formulation of the constant temperature molecular dynamics methods," The Journal of Chemical Physics 81, 511-519 (1984).

[9] William G. Hoover, "Canonical dynamics: Equilibrium phase-space distributions," Phys. Rev. A 31, 1695 (1985).

[10] Glenn J. Martyna, Michael L. Klein, and Mark Tuckerman, "Nosé-Hoover chains: The canonical ensemble via continuous dynamics," The Journal of Chemical Physics 97, 2635-2643 (1992).

[11] A Togo and I Tanaka, "First principles phonon calculations in materials science," Scr. Mater. 108, 1-5 (2015).

[12] "Appendix d - statistical errors," in Understanding Molecular Simulation (Second Edition), edited by Daan Frenkel and Berend Smit (Academic Press, San Diego, 2002) second edition ed., pp. $525-532$.

[13] Thomas R. Mattsson and Ann E. Mattsson, "Calculating the vacancy formation energy in 
metals: Pt, pd, and mo," Phys. Rev. B 66, 214110 (2002).

[14] Karin Carling, Göran Wahnström, Thomas R. Mattsson, Ann E. Mattsson, Nils Sandberg, and Göran Grimvall, "Vacancies in metals: From first-principles calculations to experimental data," Phys. Rev. Lett. 85, 3862-3865 (2000).

[15] Christoph Freysoldt, Blazej Grabowski, Tilmann Hickel, Jörg Neugebauer, Georg Kresse, Anderson Janotti, and Chris G. Van de Walle, "First-principles calculations for point defects in solids," Rev. Mod. Phys. 86, 253-305 (2014).

[16] R. Nazarov, T. Hickel, and J. Neugebauer, "Vacancy formation energies in fcc metals: Influence of exchange-correlation functionals and correction schemes," Phys. Rev. B 85, 144118 (2012).

[17] Ann E. Mattsson, Ryan R. Wixom, and Rickard Armiento, "Electronic surface error in the Si interstitial formation energy," Phys. Rev. B 77, 155211 (2008).

[18] Ann E. Mattsson, Rickard Armiento, Peter A. Schultz, and Thomas R. Mattsson, "Nonequivalence of the generalized gradient approximations PBE and PW91," Phys. Rev. B 73, 195123 (2006).

[19] J. P. Perdew, K. Burke, and M. Ernzerhof, "Generalized gradient approximation made simple." Phys. Rev. Lett. 77, 3865-3868 (1996).

[20] G. Kresse and D. Joubert, "From ultrasoft pseudopotentials to the projector augmented-wave method," Phys. Rev. B 59, 1758-1775 (1999).

[21] G. Kresse and J. Hafner, "Ab initio molecular dynamics for liquid metals," Phys. Rev. B 47, 558-561 (1993).

[22] G. Kresse and J. Furthmüller, "Efficiency of ab-initio total energy calculations for metals and semiconductors using a plane-wave basis set," Computational Materials Science 6, 15 - 50 (1996).

[23] G. Kresse and J. Furthmüller, "Efficient iterative schemes for ab initio total-energy calculations using a plane-wave basis set," Phys. Rev. B 54, 11169-11186 (1996).

[24] P. E. Blöchl, "Projector augmented-wave method," Phys. Rev. B 50, 17953-17979 (1994).

[25] G. Kresse and D Joubert, "From ultrasoft pseudopotentials to the projector augmented-wave method." Phys. Rev. B 59, 1758-1775 (1999).

[26] John P. Perdew, "Generalized gradient approximation for the fermion kinetic energy as a functional of the density," Physics Letters A 165, 79 - 82 (1992). 
